\title{
ANÁLISIS COMPARATIVO Y MODELACIÓN DE LAS SITUACIONES DE CALIDAD DEL AIRE EN UNA MUESTRA DE CIUDADES DEL MUNDO. COMPARACIÓN CON EL CASO DE MEDELLÍN.
}

\author{
COMPARATIVE ANALYSIS AND MODELLING OF AIR QUALITY SITUATIONS IN A SAMPLE OF \\ WORLD CITIES. COMPARISON WITH THE MEDELLIN CASE
}

\author{
Enrique Posada1, Miryam Gómez ${ }^{2}$, Johan Almanza ${ }^{3}$ \\ ${ }^{1}$ Asesor de proyectos especiales, HATCH INDISA S.A. Medellín-Colombia \\ enrique.posada@hatchindisa.com \\ 2 Investigadora Grupo GHYGAM, Politécnico Colombiano Jaime Isaza Cadavid. Medellín-Colombia \\ mgomez@elpoli.edu.co \\ ${ }^{3}$ Ingeniero johan.almanza1@gmail.com
}

\section{RESUMEN}

Para una muestra de 25 ciudades de diversos países del mundo, se recogió información sobre la calidad anual del aire para $\mathrm{PM}_{2.5}, \mathrm{NO}_{2}$ y ozono y sobre diversos parámetros de interés en cuanto a su relación potencial con la calidad del aire. Un análisis comparativo de dicha información y un modelo lineal que relaciona la calidad del aire con algunos de los parámetros es elaborado, con base en el nivel de aproximación que se logró entre las calidades reportadas y las predichas por la modelación. Los datos se basan en reportes e información disponible en páginas web y en reportes públicos e informes de investigación propios para los años 2012 y 2015. Se ha logrado plantear una perspectiva novedosa de la situación de la calidad del aire y del tipo de medidas estratégicas que se pudieran emprender para lograr mejorar la situación y llevarla a los límites deseables.

Palabras clave: calidad del aire, contaminantes criterio, indicadores de calidad del aire, ciudades, análisis, correlaciones, comparaciones, estrategia, Medellín, parámetros, variables ambientales.

Recibido: 24 de Abril de 2017. Aceptado: 25 de Agosto de 2017

Received: April 24, $2017 \quad$ Accepted: August 25, 2017

\begin{abstract}
For a sample of 25 cities from different countries, information was collected on the annual air quality for $P M_{2.5}, \mathrm{NO}_{2}$ and ozone and also on various parameters of interest as to their potential relation with air quality. A comparative analysis of this information and a linear model that relates the air quality to some of the parameters was elaborated, based on the level of approximation found between the air qualities reported and those predicted by the modeling. The data are based on reports and information available on websites and public reports and also on research reports done by the authors for the years 2012 and 2015. With this comparative analysis it has been possible to present a novel perspective on the air quality situation of the city and on the type of strategic measures that could be taken to improve the air quality situation
\end{abstract}

Keywords: Air quality, Criteria pollutants, Air Quality Indicators, cities, analysis, correlations, comparisons, strategy, Medellín, parameters, environmental variables.

Cómo citar este artículo: E. Posada, M. Gómez, J. Almanza, "Análisis comparativo y modelación de las situaciones de calidad del aire en una muestra de ciudades del mundo. comparación con el caso de Medellín," Revista Politécnica, vol. 13, no. 25, pp. 9-29, 2017. 


\section{INTRODUCCIÓN}

Existe una preocupación general en todo el mundo para comprender la situación de los problemas de la calidad del aire, especialmente si se considera que está bastante correlacionada con problemas de salud pública. En este sentido la Organización Mundial de la Salud y otras entidades han compilado datos de los niveles de calidad del aire, en términos de las concentraciones de diversas sustancias que se consideran de interés prioritario. Tales compilaciones aparecen en informes periódicos [ver como ejemplos 1,2,3] y se basan en los reportes de las diversas redes de medición que existen en todo el mundo, notablemente en los centros urbanos [ver como ejemplos 4, 5,6].

$\mathrm{Si}$ bien es amplia la lista de sustancias contaminantes que existen en el aire de las zonas urbanas, se han establecido por parte de las autoridades listas prioritarias por parte de las autoridades ambientales y de salud pública. De ellas se han escogido tres para este estudio: el material particulado fino de tamaño menos de 2.5 micras, conocido como $\mathrm{PM}_{2.5}$, el óxido de nitrógeno $\mathrm{NO}_{2}$ y el ozono $\mathrm{O}_{3}$.

El $\mathbf{P M}_{2.5}$ de las ciudades proviene en buena parte de procesos de combustión incompleta, mayoritariamente provenientes de los vehículos [7]. Se trata de compuestos de carbono (hollín y carbón negro) y sus derivados (carbono orgánico, compuestos de carbono, hidrógeno y oxígeno). Aportan también el desgaste de llantas y de vías y la construcción. Igualmente son significativos los impactos de reacciones secundarias originadas en óxidos de azufre y de nitrógeno, estimuladas por la luz solar y por agentes oxidantes, como el ozono; también hay presencia de compuestos derivados del amoníaco que se genera en la agricultura y en la ganadería y en su menor parte de sustancias minerales asociadas con la industria y con otras actividades humanas. En ciertas zonas hay riqueza de aerosoles asociados con el mar. En el caso de la ciudad de Medellín y el Valle de Aburra, algunos estudios realizados [8] estiman que el $\mathrm{PM}_{2.5}$ resulta de las siguientes fuentes: emisiones de vehículos y desgaste de llantas, 64,6\%; desgaste asociado con vías, 5,0\%; emisiones de calderas y combustión industrial, 8,5\%; fuentes naturales, construcciones y contaminación de fondo, $14,4 \%$ y reacciones secundarias, 7,4 \% [9].
EL $\mathrm{PM}_{2.5}$ está asociado con problemas de salud relacionados especialmente con los sistemas respiratorio y circulatorio. Las normas nacionales de Colombia [10] señalan los siguientes límites: 25 $\mathrm{\mu g} / \mathrm{m}^{3}$ (microgramos por cada metro cúbico de aire ambiental) para promedio anual y $50 \mu \mathrm{g} / \mathrm{m}^{3}$ para mediciones de 24 horas.

El $\mathrm{NO}_{2}$ hace parte de los denominados $\mathrm{NOx}$, óxidos de nitrógeno, y es el mayor componente de los mismos. Proviene ante todo de los procesos de combustión, en los cuales, dada la existencia de oxígeno y nitrógeno en el aire y las altas temperaturas, se da lugar a una pequeña oxidación del nitrógeno, en función de las condiciones de la combustión. Se estima que en el Valle de Aburrá, los vehículos aportan el $80 \%$ de los NOx [11].

Los óxidos de nitrógeno participan en reacciones atmosféricas que dan lugar a la formación de sales en el material particulado, y a la formación de ozono. En cuanto a la salud, se considera que los NOx afectan el sistema respiratorio. Las normas nacionales de Colombia [10] señalan los siguientes límites: $100 \mu \mathrm{g} / \mathrm{m}^{3}$ (microgramos por cada metro cúbico de aire ambiental) para promedio anual; 150 $\mu \mathrm{g} / \mathrm{m}^{3}$ para mediciones de 24 horas y $200 \mu \mathrm{g} / \mathrm{m}^{3}$ para mediciones de 1 hora.

El ozono, $\mathrm{O}_{3}$, es un compuesto oxidante, que resulta en su mayor parte de reacciones entre los óxidos de nitrógeno y compuestos orgánicos volátiles (que provienen de la combustión parcial o de la evaporación de combustibles o solventes orgánicos). Estas reacciones reciben soporte de la luz solar y de la temperatura. Es claro que, en las ciudades, están asociados especialmente con los vehículos.

El ozono se considera dañino para la salud, por el efecto en estas reacciones secundarias y por su carácter oxidante y reactivo, que afecta las vías respiratorias generando irritaciones. Las normas nacionales de Colombia [10] señalan los siguientes límites: $80 \mu \mathrm{g} / \mathrm{m}^{3}$ (microgramos por cada metro cúbico de aire ambiental) para promedio de 8 horas y $120 \mu \mathrm{g} / \mathrm{m}^{3}$ para mediciones de 1 hora.

Como se puede apreciar en las consideraciones anteriores, las ciudades constituyen sitios de interés prioritario para estudiar los contaminantes escogidos, dado que el tráfico automotor es la fuente más importante de la problemática. Además, las autoridades ambientales concentran en las 
ciudades, no solamente las redes de medición de la contaminación, sino los sistemas de toma de información meteorológica. Por ello se ha decidido hacer el análisis para diversos centros urbanos de tamaño variado y de distintas características distribuidos por el mundo.

\section{MATERIALES Y METODOS}

Se escogieron 25 ciudades para el análisis. Se tuvo en cuenta escoger ciudades tanto de países desarrollados como de países en desarrollo; de ciudades costeras y de ciudades del interior; de ciudades situadas en zonas montañosas y de ciudades en zonas planas; de ciudades muy populosas y de ciudades de menor población. La muestra se seleccionó de manera que se contara con información suficiente para establecer las comparaciones. Se escogieron varias ciudades de Latinoamérica y dos de Colombia. Para cada una de ellas se llevó a cabo una búsqueda de información sobre las concentraciones anuales de $\mathrm{PM}_{2.5}, \mathrm{NO}_{2}$ y ozono y una búsqueda de datos de diversos parámetros que se consideraban de interés en cuanto a su relación potencial con la calidad del aire.

Hay que advertir que no es fácil encontrar la información ambiental para las distintas ciudades y llevarla a bases comparativas. Por una parte, en general se cuenta en cada ciudad con una red de estaciones de calidad del aire, entre las cuales se encuentran variaciones significativas en los datos, tanto temporales como espaciales. Organizaciones diversas presentan algunos resúmenes consolidados, pero no está disponible en todos los casos, la trazabilidad y la metodología para encontrar valores representativos de los promedios anuales de las concentraciones. Este estudio que presentamos puede adolecer igualmente de estos problemas y por ello se trató de revisar datos reportados según aparecen en páginas de la web, tratando de consultar varias fuentes y de ir directamente a los datos de las estaciones cuando estos estaban disponibles. El contaminante que ofrece las mayores dificultades de análisis comparativo es el ozono, para el cual no fue factible encontrar en todos los casos información sobre promedios anuales, además de que no se mide con tanta abundancia y número de estaciones como el $\mathrm{PM}_{2.5}$ y el $\mathrm{NO}_{2}$. Hay que decir que es también el contaminante para el cual este estudio encontró las mayores dificultades de modelación.

En cuanto a los parámetros que se estudiaron, se escogieron los que se describen en la tabla 1. El criterio para seleccionar esta lista es de los autores y está basado en su experiencia académica e investigativa. La revisión que se hizo de literatura muestra que es un asunto que se ha trabajado muy poco en términos comparativos entre ciudades. Se trató de trabajar con parámetros que tuvieran relación con la actividad urbana y con aquello que hace distintas a las ciudades del resto de las zonas de una región, bajo el supuesto de que la actividad urbana de cada zona es, en última instancia, la que da origen a su propia contaminación. No obstante, lo anterior, es evidente de los datos y de su análisis comparativo, que existe una contaminación base no directamente atribuible a las ciudades o a los parámetros que fueron seleccionados para caracterizar tales ciudades.

Tabla 1. Parámetros estudiados para modelar la calidad del aire comparativa

\begin{tabular}{lll}
\hline Parámetro & unidades & \multicolumn{1}{c}{ Notas e hipótesis de trabajo } \\
\hline 1. Habitantes & millones & $\begin{array}{l}\text { Son los habitantes de la zona urbana considerada. En } \\
\text { general se tomaron las zonas metropolitanas. Acá hay } \\
\text { una amplia riqueza de datos. Se tuvo cuidado en que el } \\
\text { dato escogido fuera consistente con los demás } \\
\text { parámetros. }\end{array}$ \\
\hline 2. Área urbana & $\mathrm{Km}^{2}$ & $\begin{array}{l}\text { Depende altamente de la zona considerada. Se utilizaron } \\
\text { valores que fueran consistentes con los demás } \\
\text { parámetros. En todos los casos se refinó el análisis con } \\
\text { las manchas urbanas como se observan en los mapas de } \\
\text { google maps. }\end{array}$ \\
&
\end{tabular}




\begin{tabular}{lll}
\hline \multicolumn{1}{c}{ Parámetro } & unidades & \multicolumn{1}{c}{ Notas e hipótesis de trabajo } \\
\hline $\begin{array}{l}\text { 3. Densidad } \\
\text { poblacional }\end{array}$ & Habitantes $/ \mathrm{km}^{2}$ & $\begin{array}{l}\text { Se obtiene al combinar (1) y (2). Se lo considera como } \\
\text { uno de los claros indicadores de actividad humana y } \\
\text { como relacionado con la calidad del aire. }\end{array}$ \\
\hline
\end{tabular}

4. Carros Miles

Vehículos particulares en la zona urbana considerada. Es complejo recoger esta información, dadas las distintas metodologías de registro que existen y las claras variaciones en el tiempo.

\begin{tabular}{llll}
\hline 5. Motos & Miles & $\begin{array}{l}\text { Motos (2 ruedas) en la zona urbana considerada. Es } \\
\text { complejo recoger esta información, dadas las distintas } \\
\text { metodologías de registro que existen y las claras } \\
\text { variaciones en el tiempo. }\end{array}$ \\
\hline $\begin{array}{l}\text { 6.Densidad } \\
\text { poblacional } \\
\text { carros }\end{array}$ & de & Carros/mil habitantes & Se obtiene al combinar (4) y (1). \\
\hline $\begin{array}{l}\text { Densidad } \\
\text { poblacional } \\
\text { motos }\end{array}$ & de & Motos $/$ mil habitantes & Se obtiene al combinar (5) y (1). \\
\hline
\end{tabular}

\begin{tabular}{|c|c|}
\hline $\begin{array}{l}\text { 8. Densidad } \\
\text { poblacional } \\
\text { vehicular }\end{array}$ & $\begin{array}{c}\text { Carros equivalentes/mil } \\
\text { habitantes }\end{array}$ \\
\hline
\end{tabular}

9. Densidad
urbana vehicular $\quad$ Carros equivalentes $/ \mathrm{km}^{2}$

9. Densidad

urato

\begin{tabular}{lll} 
10. Área vehicular & \% Área urbana & $\begin{array}{l}\text { Se obtiene al combinar (4), (5) y (2) Se ha considerado } \\
\text { que cada carro ocupa 6.3 m2 y cada moto } 1.2 \text { m2 }\end{array}$ \\
$\begin{array}{c}\text { 11. Accidentes de Miles de accidentes por } \\
\text { año }\end{array}$ & $\begin{array}{l}\text { Es complejo recoger esta información, dadas las distintas } \\
\text { metodologías de registro que existen y las claras } \\
\text { variaciones en el tiempo. Se tomaron los accidentes } \\
\text { totales que se reportan. }\end{array}$ \\
\hline
\end{tabular}
cada moto equivale a 0.25 carros. Es uno de los indicadores de actividad vehicular, relacionado con la calidad del aire.

Se obtiene al combinar (4), (5) y (2) Se ha considerado cada carro ocupa 6.3 m2 y cada moto $1.2 \mathrm{~m} 2$ que se reportan.
Se obtiene al combinar (6) y (7), Se consideró que cada moto equivale a 0.25 carros. Es uno de los indicadores de actividad vehicular, relacionado con la calidad del aire.

Se obtiene al combinar (8) y (1) y (2) considerando que

$\begin{array}{ll}\begin{array}{l}\text { 12. Accidentabilidad } \\ \text { vehicular }\end{array} & \begin{array}{l}\text { Accidentes por cada mil } \\ \text { vehículos equivalentes }\end{array}\end{array}$

Se obtiene al combinar (11), (4) y (5) considerando que cada moto equivale a 0.25 carros. Se lo considera como uno de los indicadores de actividad vehicular y de cultura, relacionado con la calidad del aire

\begin{tabular}{lll}
\hline $\begin{array}{l}\text { 13. Riqueza } \\
\text { (Ingreso per cápita) }\end{array}$ & US \$/persona año & $\begin{array}{l}\text { Se lo considera como uno de los indicadores de } \\
\text { actividad urbana, de cultura y de desarrollo, relacionado } \\
\text { con la calidad del aire de forma compleja }\end{array}$ \\
\hline $\begin{array}{l}\text { 14. Nivel } \\
\text { urbana }\end{array}$ zona & m sobre el nivel del mar & $\begin{array}{l}\text { Con la ayuda de google earth, se obtuvieron promedios } \\
\text { de la altura sobre el nivel del mar en la zona urbana }\end{array}$ \\
\hline $\begin{array}{l}\text { 15. Nivel zona que } \\
\text { limita a la zona } \\
\text { urbana }\end{array}$ & m sobre el nivel del mar & $\begin{array}{l}\text { La zona que la limita, se obtuvo considerándola en } \\
\text { promedio como } 3,4 \text { veces la urbana (con variaciones } \\
\text { según casos entre } 2,6 \text { y } 4,4 \text { veces). }\end{array}$ \\
\hline
\end{tabular}




\begin{tabular}{lll}
\hline \multicolumn{1}{c}{ Parámetro } & unidades & \multicolumn{1}{c}{ Notas e hipótesis de trabajo } \\
\hline $\begin{array}{l}\text { 16. Topografía de la } \\
\text { zona urbana }\end{array}$ & Factor de desnivel $(\mathrm{m})$ & $\begin{array}{l}\text { El factor de desnivel se obtiene restando el nivel de la } \\
\text { zona que limita menos el nivel de la zona urbana }(15)- \\
(14) \text {. Se lo considera un indicador de la complejidad de la } \\
\text { topografía, el cual se espera que impacte la calidad del } \\
\text { aire }\end{array}$ \\
\hline $\begin{array}{l}\text { 17. Velocidad del } \\
\text { viento media anual }\end{array}$ & $\mathrm{Km} / \mathrm{hr}$ & $\begin{array}{l}\text { Promedio anual. Es complejo recoger la información de } \\
\text { clima (17), (18), (19) dadas las distintas metodologías de } \\
\text { registro que existen y las variaciones en el tiempo y en el } \\
\text { espacio. Se la considera como influyente en la calidad del } \\
\text { aire }\end{array}$ \\
\hline $\begin{array}{l}\text { 18. Nubosidad anual } \\
\text { media }\end{array}$ & Ver nota anterior \\
\hline $\begin{array}{l}\text { 19. Lluvia media } \\
\text { anual }\end{array}$ & Precipitación anual $(\mathrm{mm})$ & Ver nota anterior \\
\hline
\end{tabular}

Recogidos los datos, revisados, puestos en bases coherentes, se realizó un análisis comparativo de dicha información. En general se trató de comparar los parámetros de calidad del aire y de influencia con la situación particular de la ciudad de Medellín, dado que los autores de este trabajo viven en el área y el mismo está en buena parte dirigido a establecer perspectivas y conclusiones locales, pero sin perder la visión integrada y universal de los fenómenos estudiados, sobre todo si se tiene en cuenta que hacia el futuro la ciudad de Medellín irá evolucionando en algunas de las direcciones mostradas por las distintas ciudades de la muestra o alejándose cada vez más, en aquellos casos en que la ciudad muestra características muy propias y particulares.

Dado que se propusieron variables hipotéticamente influyentes en la calidad del aire, como las señaladas en la tabla 1, resulta natural que se intente la modelación de las situaciones de las distintas ciudades, tratando de encontrar correlaciones y factores de influencia de naturaleza lo más universal que sea posible, sin que se pretenda, en esta etapa del estudio comparativo, establecer relaciones de causalidad. Para ello se elaboró un modelo lineal que relaciona la calidad del aire con algunos de los parámetros, los cuales fueron escogidos con base en el nivel de aproximación que se logró entre las calidades reportadas y las predichas por la modelación. Se ha logrado con base en este análisis contar con una perspectiva novedosa de la situación de la calidad del aire y del tipo de medidas que hay que tomar para lograr ajustarla a valores dentro de los límites deseables.

\section{RESULTADOS Y ANÁLISIS COMPARATIVO}

Las tablas 2, 3, 4, y 5 muestran los resultados obtenidos luego de las búsquedas realizadas para todos los parámetros. Dada la riqueza de datos, para una mejor visualización comparativa, se han organizado por categorías de tipo ambiental, tabla 2; de tipo demográfico, área y geográfico, tabla 3; de actividad y de vehículos, tabla 4 y tabla 5 de tipo climático.

En la tabla 2 se ha estimado un índice combinado ambiental y un índice de calidad con base en la siguiente expresión que compara los datos anuales con las normas aplicables:

$$
\begin{gathered}
I A=\text { ConcPM2.5/NPM } 2.5 \times 0,50+ \\
\begin{array}{c}
\text { ConcNO } \\
2
\end{array} \mathrm{NNO}_{2} \times 0,25+ \\
\text { ConcOzono } / \text { Nozono } \times 0,25 \\
\mathrm{IC}=1 / \mathrm{A} \quad \text { (2) }
\end{gathered}
$$

Donde:

$$
\begin{gathered}
\mathrm{NPM} 2.5=25 \mu \mathrm{g} / \mathrm{m}^{3} ; \mathrm{NNO}_{2}=100 \mu \mathrm{g} / \mathrm{m}^{3} ; \\
\text { Nozono }=60 \mu \mathrm{g} / \mathrm{m}^{3}
\end{gathered}
$$

Para el ozono el valor de Nozono ha sido escogido por los autores ya que no se especifica la norma anual. Para ello se ha tomado la norma de 1 hora extrapolada a 24 horas, siguiendo las proporciones de las normas del $\mathrm{NO}_{2}$

A mayor IC, mejor la calidad del aire; a mayor IA, menor la calidad del aire. 
Tabla 2. Datos anuales promedio de calidad del aire e indicadores ambientales y de calidad (CR)

\begin{tabular}{|c|c|c|c|c|c|c|c|c|}
\hline Ciudad & $\begin{array}{c}\mathrm{PM}_{2.5} \\
\mu \mathrm{gr} / \mathrm{m}^{3}\end{array}$ & $\begin{array}{c}\mathrm{NO}_{2}, \\
\mu \mathrm{gr} / \mathrm{m}^{3}\end{array}$ & $\begin{array}{l}\text { Ozono, } \\
\mu \mathrm{gr} / \mathrm{m}^{3}\end{array}$ & $\mathrm{IAPM}_{2,5}$ & $\mathrm{IANO}_{2}$ & $\mathrm{IAO}_{3}$ & IA & IC \\
\hline Amsterdam & 15,5 & 26,0 & 32,6 & 0,62 & 0,26 & 0,54 & 0,51 & 1,968 \\
\hline Beijing & 55,6 & 57,0 & 70,0 & 2,22 & 0,57 & 1,17 & 1,55 & 0,647 \\
\hline Bogotá & 24,3 & 40,0 & 19,4 & 0,97 & 0,40 & 0,32 & 0,67 & 1,502 \\
\hline Cochabamba & 41,0 & 30,6 & 62,4 & 1,64 & 0,31 & 1,04 & 1,16 & 0,865 \\
\hline Denver-Aurora & 10,0 & 47,0 & 78,1 & 0,40 & 0,47 & 1,30 & 0,64 & 1,556 \\
\hline Guadalajara & 28,2 & 57,2 & 78,0 & 1,13 & 0,57 & 1,30 & 1,03 & 0,969 \\
\hline Hong Kong & 26,7 & 64,1 & 39,0 & 1,07 & 0,64 & 0,65 & 0,86 & 1,168 \\
\hline Houston & 10,3 & 31,6 & 51,4 & 0,41 & 0,32 & 0,86 & 0,50 & 2,004 \\
\hline Yakarta & 21,0 & 37,6 & 21,0 & 0,84 & 0,38 & 0,35 & 0,60 & 1,662 \\
\hline Juárez & 18,5 & 26,3 & 46,3 & 0,74 & 0,26 & 0,77 & 0,63 & 1,590 \\
\hline La Paz & 23,0 & 40,3 & 31,9 & 0,92 & 0,40 & 0,53 & 0,69 & 1,442 \\
\hline León & 24,1 & 45,5 & 68,9 & 0,96 & 0,46 & 1,15 & 0,88 & 1,134 \\
\hline Lima & 31,0 & 36,0 & 35,0 & 1,24 & 0,36 & 0,58 & 0,86 & 1,168 \\
\hline Los Ángeles & 12,6 & 50,8 & 98,3 & 0,50 & 0,51 & 1,64 & 0,79 & 1,268 \\
\hline Madrid & 10,2 & 35,0 & 51,5 & 0,41 & 0,35 & 0,86 & 0,51 & 1,978 \\
\hline Manila & 22,5 & 28,0 & 68,7 & 0,90 & 0,28 & 1,15 & 0,81 & 1,240 \\
\hline Medellín & 26,9 & 36,5 & 31,0 & 1,08 & 0,37 & 0,52 & 0,76 & 1,319 \\
\hline México & 26,2 & 54,2 & 59,4 & 1,05 & 0,54 & 0,99 & 0,91 & 1,103 \\
\hline Monterrey & 25,9 & 29,0 & 55,2 & 1,04 & 0,29 & 0,92 & 0,82 & 1,219 \\
\hline Montevideo & 20,3 & 25,1 & 41,8 & 0,81 & 0,25 & 0,70 & 0,64 & 1,554 \\
\hline Quito & 23,3 & 23,3 & 44,1 & 0,93 & 0,23 & 0,74 & 0,71 & 1,412 \\
\hline San Juan (AMSJ) & 9,3 & 37,6 & 84,0 & 0,37 & 0,38 & 1,40 & 0,63 & 1,587 \\
\hline Santiago de Chile & 26,0 & 41,0 & 28,8 & 1,04 & 0,41 & 0,48 & 0,74 & 1,347 \\
\hline Sao Paulo & 20,3 & 39,9 & 36,0 & 0,81 & 0,40 & 0,60 & 0,66 & 1,525 \\
\hline Tokio & 13,0 & 33,9 & 62,8 & 0,52 & 0,34 & 1,05 & 0,61 & 1,649 \\
\hline Promedio & 22,6 & 38,9 & 51,8 & 0,90 & 0,39 & 0,86 & 0,77 & 1,395 \\
\hline Medellín/promedio & 1,19 & 0,94 & 0,60 & 1,19 & 0,94 & 0,60 & 0,99 & 0,946 \\
\hline
\end{tabular}

La figura 1 muestra los datos de concentración de $\mathrm{PM}_{2.5}$. La figura 2 muestra los índices ambientales calculados. Se observan rangos relativamente amplios en los datos de calidad, especialmente en el caso del $\mathrm{PM}_{2.5}$, que es el contaminante más cercano, en promedio, en superar los límites ambientales establecidos. De todas formas, la "ciudad promedio" cumple las normas. Medellín está bastante cercano, desde el punto de vista del índice combinado ambiental, al comportamiento promedio. Su calidad es mejor que el promedio en $\mathrm{NO}_{2}$ y en Ozono e inferior en $\mathrm{PM}_{2.5}$, en el cual muestra valores un $8 \%$ sobre la norma y un $19 \%$ sobre la ciudad promedio. Hay que anotar que los datos de Medellín y del Valle de Aburrá aparecen reportados anualmente por el AMVA [12]

La tabla 3 muestra los parámetros demográficos, de área y geográficos. Se observa que hay rangos muy amplios en estos parámetros. Medellín es una de las ciudades menos pobladas de la muestra, muestra de ciudades que tiene 5,4 millones de personas en promedio. Medellín tiene una de las mayores densidades poblacionales y es la que mayor desnivel de alturas tiene entre las zonas urbanas y las zonas vecinas, al estar situada en un valle profundo. 


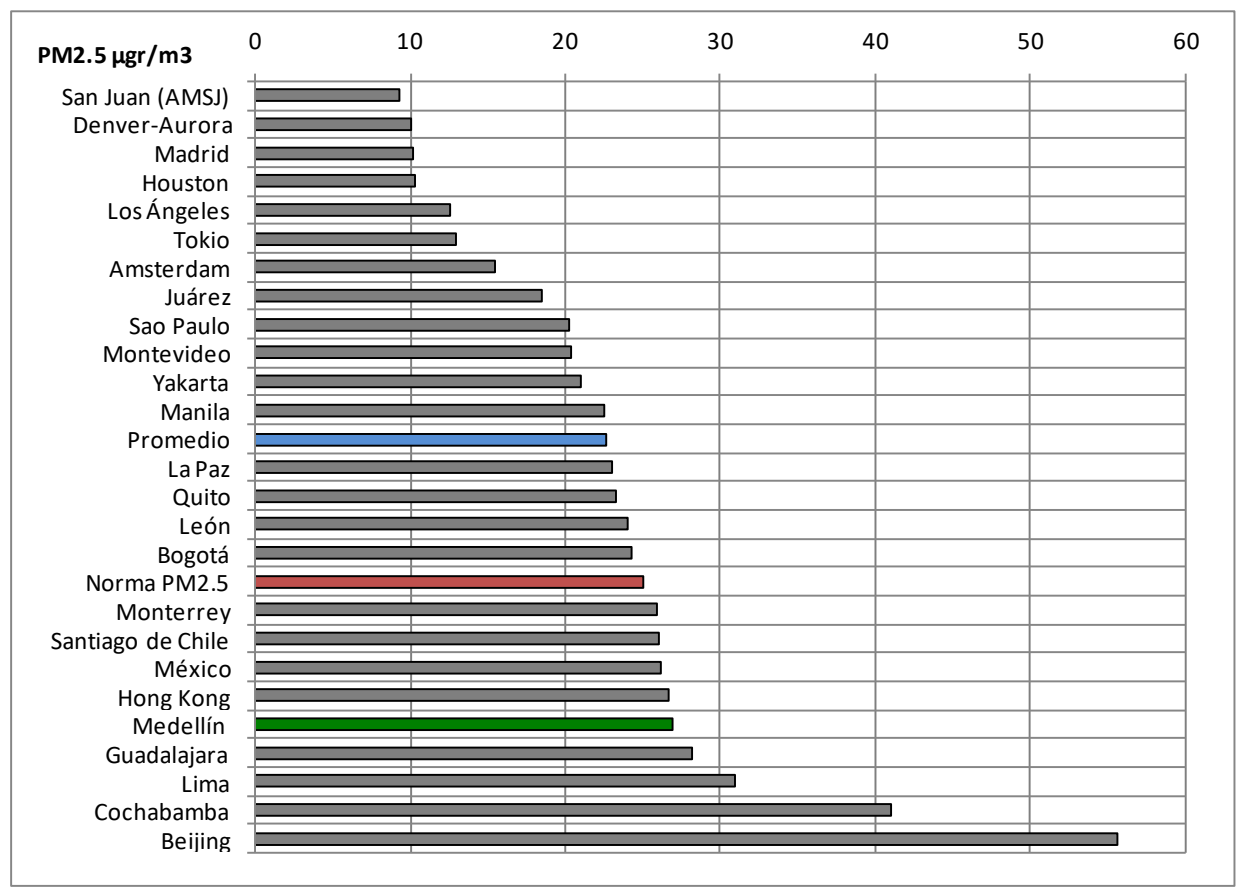

Fig. 1. Datos de $\mathrm{PM}_{2.5}$ ordenados por concentración media anual

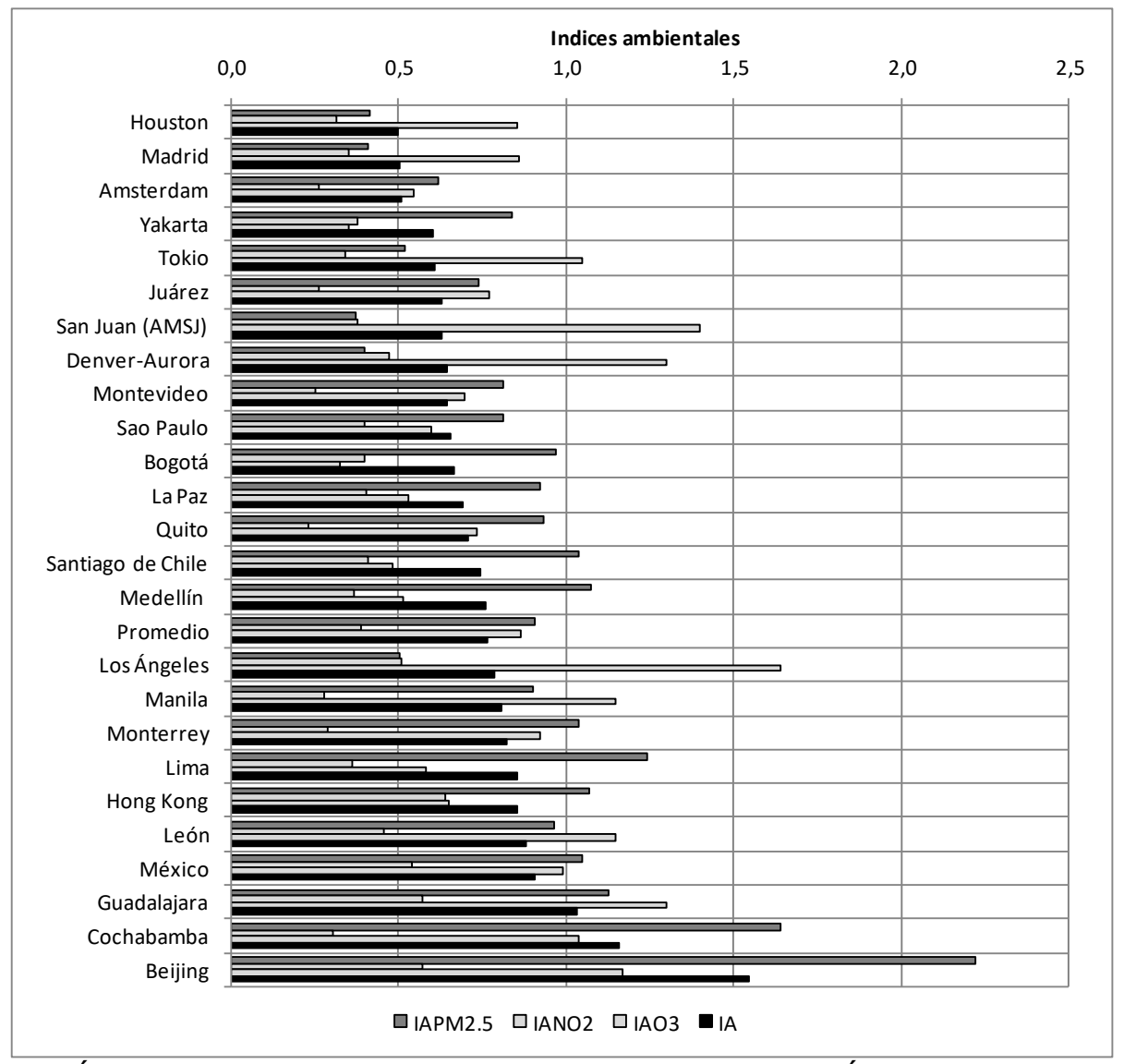

Fig.2. Índices de contaminación ambientales ordenados por Índice combinado IA 
Tabla 3. Parámetros demográficos y geográficos

\begin{tabular}{|c|c|c|c|c|c|c|}
\hline Ciudad & $\begin{array}{l}\text { habitantes } \\
\text { millones }\end{array}$ & $\begin{array}{c}\text { Área } \\
\text { urbana, } \\
\mathrm{Km}^{2}\end{array}$ & $\begin{array}{c}\text { Densidad, } \\
\text { hab/km² }\end{array}$ & $\begin{array}{c}\text { Altura s.n.m. } \\
\text { Área Urbana, } \\
\text { m }\end{array}$ & $\begin{array}{c}\text { Altura } \\
\text { s.n.m. } \\
\text { Área } \\
\text { Vecina, m }\end{array}$ & $\begin{array}{c}\text { Desnível, } \\
\text { m }\end{array}$ \\
\hline Amsterdam & 0,81 & 165 & 4.909 & -3 & 1 & 4 \\
\hline Beijing & 21,52 & 1.583 & 13.589 & 47 & 152 & 105 \\
\hline Bogotá & 7,88 & 337 & 23.404 & 2.560 & 2.752 & 192 \\
\hline $\begin{array}{l}\text { Cochabamb } \\
\text { a }\end{array}$ & 0,67 & 87 & 7.705 & 2.614 & 2.828 & 214 \\
\hline $\begin{array}{l}\text { Denver- } \\
\text { Aurora }\end{array}$ & 2,37 & 1.522 & 1.560 & 1.671 & 1.912 & 241 \\
\hline Guadalajara & 1,56 & 151 & 10.358 & 1.605 & 1.627 & 22 \\
\hline Hong Kong & 7,32 & 221 & 33.172 & 14 & 171 & 157 \\
\hline Houston & 2,24 & 1.584 & 1.414 & 15 & 24 & 9 \\
\hline Yakarta & 10,18 & 449 & 22.683 & 13 & 26 & 13 \\
\hline Juárez & 1,33 & 188 & 7.086 & 1.160 & 1.232 & 72 \\
\hline La Paz & 0,76 & 142 & 5.377 & 3.824 & 4.030 & 207 \\
\hline León & 1,58 & 198 & 7.973 & 1.838 & 1.920 & 82 \\
\hline Lima & 8,85 & 800 & 11.065 & 155 & 447 & 291 \\
\hline Los Ángeles & 3,93 & 1.200 & 3.275 & 58 & 312 & 255 \\
\hline Madrid & 3,14 & 606 & 5.187 & 660 & 652 & -8 \\
\hline Manila & 11,86 & 611 & 19.404 & 18 & 13 & -4 \\
\hline Medellín & 2,42 & 102 & 23.676 & 1.574 & 2.025 & 452 \\
\hline México & 9,05 & 1.484 & 6.098 & 2.317 & 2.668 & 351 \\
\hline Monterrey & 1,14 & 325 & 3.496 & 549 & 825 & 276 \\
\hline Montevideo & 1,72 & 194 & 8.863 & 29 & 18 & -11 \\
\hline Quito & 2,49 & 372 & 6.688 & 2.809 & 2.773 & -36 \\
\hline $\begin{array}{l}\text { San Juan } \\
\text { (AMSJ) }\end{array}$ & 1,80 & 583 & 3.093 & 22 & 18 & -4 \\
\hline $\begin{array}{l}\text { Santiago de } \\
\text { Chile }\end{array}$ & 6,16 & 641 & 9.607 & 610 & 726 & 116 \\
\hline Sao Paulo & 11,90 & 1.523 & 7.811 & 771 & 792 & 21 \\
\hline Tokio & 13,51 & 2.188 & 6.174 & 21 & 137 & 116 \\
\hline Promedio & 5,4 & 690 & 10.147 & 998 & 1.123 & 125 \\
\hline $\begin{array}{l}\text { Medellín vs } \\
\text { media }\end{array}$ & 0,44 & 0,15 & 2,33 & 1,58 & 1,80 & 3,60 \\
\hline
\end{tabular}

De nuevo, se observa que hay rangos muy amplios en estos parámetros. Medellín es una ciudad de ingresos relativamente bajos en la muestra, pero de mucha actividad vehicular. Es de las que tiene mayor ocupación de su área por vehículos, mayor concentración de motos y de las de mayor accidentabilidad. Son notables las diferencias de accidentabilidad entre las ciudades de los países desarrollados (son bajas) y las de las ciudades de países en desarrollo (son altas).

Resulta evidente que Medellín tiene concentraciones de motos, de vehículos y de accidentes bastante altas, superiores a lo que es común en las ciudades estudiadas, y ello implica que es una ciudad con grandes desafíos de tipo normativo, cultural y educativo, mayores que las de casi todas las ciudades. Habrá que hacer grandes esfuerzos e inversiones en amueblamiento urbano, infraestructura, educación y sistemas inteligentes de información y de regulación del tráfico.

El alto porcentaje del área de esta ciudad ocupada por los vehículos, fenómeno que sigue en aumento, y el hecho de que está situada en un valle estrecho indica que se requieren sistemas elevados 0 subterráneos de circulación y de parqueo y un esfuerzo continuado de movilidad colectiva de alta calidad.

La figura 3 ilustra tres de los parámetros anteriores. Para ello se ha utilizado un valor relativo, dividiendo cada parámetro por el promedio de la muestra. 
Tabla 4. Parámetros de actividad y de vehículos.

\begin{tabular}{|c|c|c|c|c|c|c|c|c|}
\hline Ciudad & $\begin{array}{l}\text { IPC } \\
\text { año, } \\
\text { US \$ }\end{array}$ & $\begin{array}{c}\text { Carros } \\
\text { /mil } \\
\text { hab. }\end{array}$ & $\begin{array}{c}\text { Motos/ } \\
\text { mil } \\
\text { hab. }\end{array}$ & $\begin{array}{l}\text { Carros } \\
\text { emú. } \\
\text { Por mil } \\
\text { hab. }\end{array}$ & $\begin{array}{c}\text { Carros } \\
\text { equ. } \\
\text { por } \\
\text { km2 }\end{array}$ & $\begin{array}{c}\text { Carros } \\
\text { y } \\
\text { motos, } \\
\% \text { área }\end{array}$ & $\begin{array}{c}\text { Acci- } \\
\text { dentes } \\
\text { por mil } \\
\text { hab. }\end{array}$ & $\begin{array}{c}\text { Acci- } \\
\text { dentes } \\
\text { por mil } \\
\text { carros } \\
\text { equ. }\end{array}$ \\
\hline Amsterdam & 45.270 & 324 & 80 & 344 & 1.690 & 1,11 & 2,10 & 6,10 \\
\hline Beijing & 23.383 & 231 & 20 & 236 & 3.206 & 2,05 & 6,58 & 27,89 \\
\hline Bogotá & 17.493 & 197 & 62 & 213 & 4.980 & 3,31 & 4,27 & 20,09 \\
\hline Cochabamba & 6.530 & 223 & 84 & 244 & 1.881 & 1,26 & 5,66 & 23,17 \\
\hline Denver-Aurora & 61.544 & 752 & 45 & 763 & 1.190 & 0,76 & 2,22 & 2,91 \\
\hline Guadalajara & 17.068 & 372 & 49 & 384 & 3.976 & 2,57 & 12,44 & 32,41 \\
\hline Hong Kong & 57.245 & 78 & 9 & 80 & 2.649 & 1,71 & 2,20 & 27,50 \\
\hline Houston & 75.000 & 938 & 48 & 950 & 1.344 & 0,85 & 8,71 & 9,16 \\
\hline Yakarta & 9.975 & 248 & 517 & 378 & 8.563 & 6,80 & 4,44 & 11,76 \\
\hline Juárez & 14.331 & 313 & 4 & 314 & 2.224 & 1,40 & 3,96 & 12,60 \\
\hline La Paz & 6.530 & 236 & 28 & 244 & 1.309 & 0,84 & 21,21 & 87,10 \\
\hline León & 15.204 & 292 & 24 & 298 & 2.374 & 1,52 & 3,42 & 11,47 \\
\hline Lima & 16.495 & 130 & 10 & 133 & 1.470 & 0,94 & 6,13 & 46,12 \\
\hline Los Ángeles & 65.053 & 1.104 & 28 & 1.111 & 3.639 & 2,30 & 7,39 & 6,65 \\
\hline Madrid & 39.280 & 978 & 78 & 997 & 5.173 & 3,31 & 4,80 & 4,81 \\
\hline Manila & 14.152 & 84 & 118 & 113 & 2.199 & 1,66 & 8,06 & 71,16 \\
\hline Medellín & 16.637 & 244 & 240 & 304 & 7.199 & 5,22 & 18,79 & 61,78 \\
\hline México & 19.212 & 489 & 23 & 494 & 3.015 & 1,92 & 3,61 & 7,31 \\
\hline Monterrey & 28.111 & 486 & 13 & 489 & 1.711 & 1,08 & 29,26 & 59,78 \\
\hline Montevideo & 24.419 & 198 & 257 & 262 & 2.324 & 1,74 & 4,90 & 18,70 \\
\hline Quito & 14.185 & 56 & 36 & 65 & 437 & 0,30 & 1,84 & 28,21 \\
\hline San Juan (AMSJ) & 17.351 & 528 & 7 & 530 & 1.640 & 1,04 & 19,31 & 36,42 \\
\hline Santiago de Chile & 23.869 & 185 & 11 & 188 & 1.807 & 1,15 & 4,20 & 22,35 \\
\hline Sao Paulo & 20.626 & 548 & 87 & 570 & 4.455 & 2,89 & 17,21 & 30,18 \\
\hline Tokio & 43.703 & 229 & 35 & 238 & 1.467 & 0,95 & 1,57 & 6,60 \\
\hline Promedio & 27.707 & 379 & 77 & 398 & 2.877 & 1,95 & 8,17 & 26,89 \\
\hline $\begin{array}{l}\text { Medellín, } \\
\text { media }\end{array}$ & 0,60 & 0,64 & 3,13 & 0,76 & 2,50 & 2,68 & 2,30 & 2,30 \\
\hline
\end{tabular}

La tabla 5 muestra los parámetros climáticos de la muestra de ciudades estudiadas. En este caso las variaciones son menos amplias.

Se observa que Medellín es una de las ciudades más lluviosas y más nubladas y es la menos ventilada de todas las de la muestra, siendo este último un factor que parece contribuir al aumento en las concentraciones PM2.5 ocasionada tanto por las mayores emisiones como por eventos meteorológicos de inversión térmica y subsidencia de masas de aire, presentados durante los últimos años. Esto es evidente desde el comportamiento de los fenómenos de dispersión atmosférica [13].

\subsection{MODELACIÓN Y CORRELACIONES ENTRE LA CALIDAD DEL AIRE $Y$ LOS PARÁMETROS CARACTERÍSTICOS DE LAS CIUDADES}

Una de las primeras inquietudes que surge al examinar la información obtenida es examinar en qué medida están relacionados entre sí los datos mismos de calidad del aire. El conjunto de gráficos de la figura 4 muestra relaciones para las influencias de los $\mathrm{NO}_{2}$ en el $\mathrm{PM}_{2.5}$ y el ozono. No se aprecia correlación significativa, si bien se nota una ligera tendencia a que ambos son mayores cuando el $\mathrm{NO}_{2}$ se incrementa. 


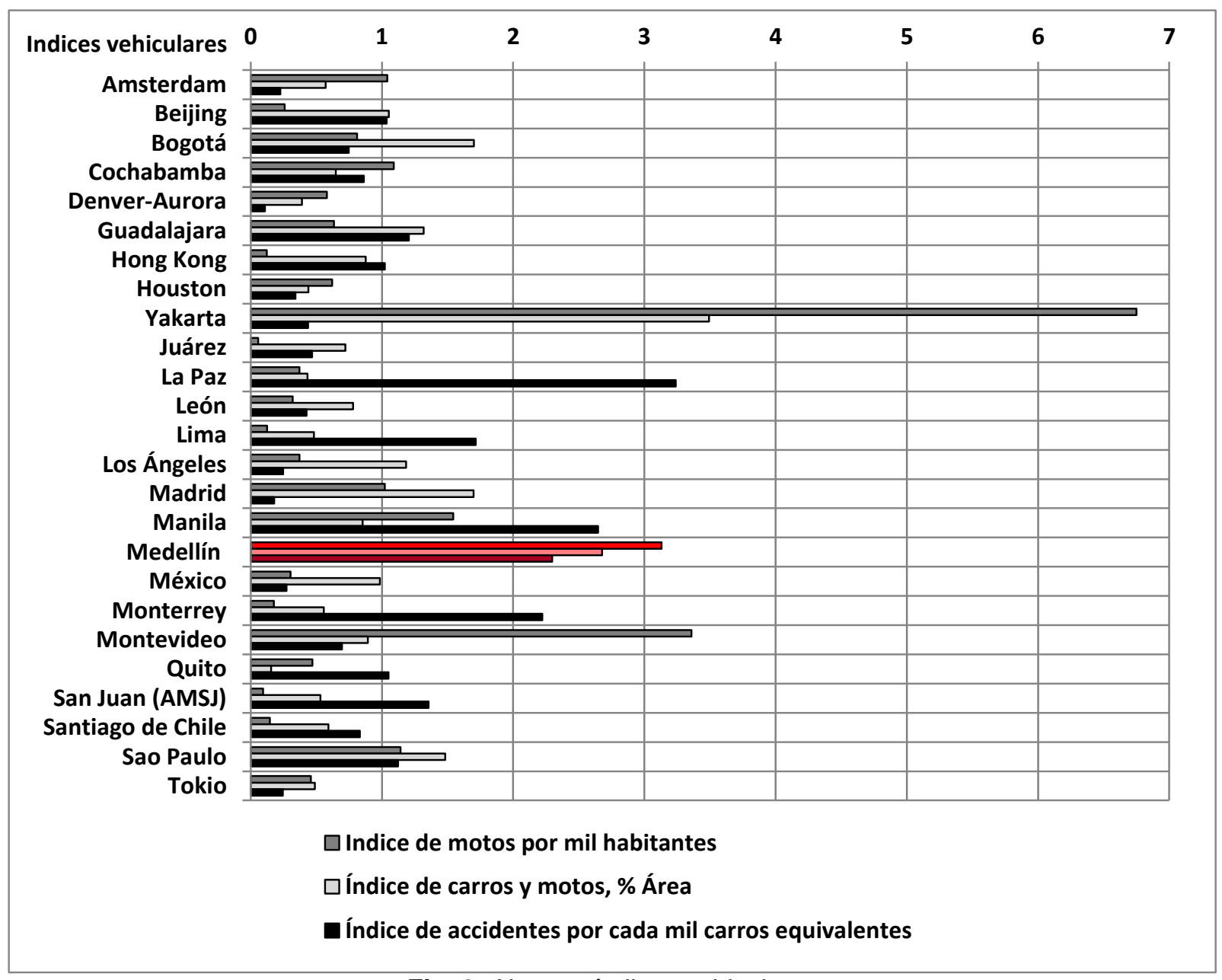

Fig. 3. Algunos índices vehiculares
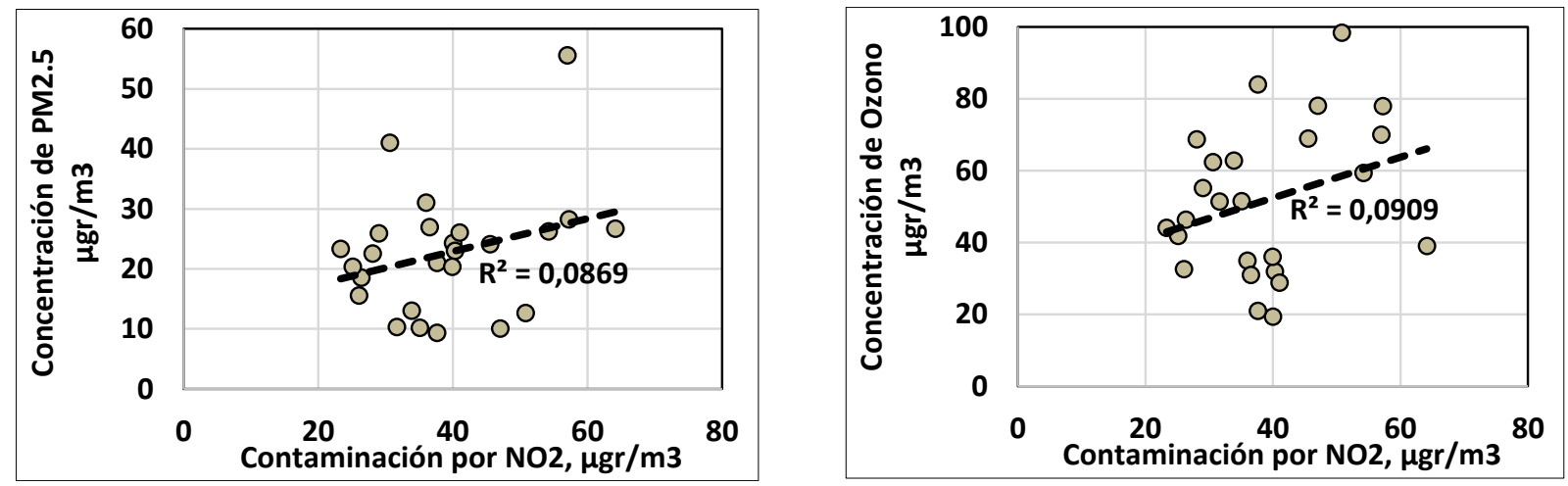

Fig. 4. Relaciones entre $\mathrm{PM}_{2.5}$, Ozono y $\mathrm{NO}_{2}$ en la muestra de ciudades estudiada 
Tabla 5. Parámetros climáticos

\begin{tabular}{|c|c|c|c|c|}
\hline Ciudad & $\begin{array}{l}\text { PM2.5 promedio } \\
\text { anual } \mu \mathrm{gr} / \mathrm{m}^{3}\end{array}$ & $\begin{array}{c}\text { Velocidad del } \\
\text { viento, media } \\
\text { anual, } \mathrm{km} / \mathrm{h} \\
\end{array}$ & $\begin{array}{l}\text { Nubosidad media } \\
\text { anual (\%) }\end{array}$ & $\begin{array}{c}\text { Precipitación de } \\
\text { Iluvias anuales } \\
(\mathrm{mm})\end{array}$ \\
\hline Amsterdam & 15,5 & 9,3 & 75,0 & 766 \\
\hline Beijing & 55,6 & 9,0 & 40,0 & 587 \\
\hline Bogotá & 24,3 & 7,9 & 61,0 & 991 \\
\hline Cochabamba & 41,0 & 9,7 & 46,0 & 752 \\
\hline Denver-Aurora & 10,0 & 14,0 & 65,0 & 395 \\
\hline Guadalajara & 28,2 & 11,3 & 45,0 & 871 \\
\hline Hong Kong & 26,7 & 16,1 & 55,0 & 1.949 \\
\hline Houston & 10,3 & 12,2 & 77,0 & 1.156 \\
\hline Yakarta & 21,0 & 9,7 & 67,5 & 1.872 \\
\hline Juárez & 18,5 & 11,5 & 30,0 & 308 \\
\hline La Paz & 23,0 & 9,0 & 50,0 & 484 \\
\hline León & 24,1 & 11,3 & 50,0 & 622 \\
\hline Lima & 31,0 & 11,9 & 75,0 & 0 \\
\hline Los Ángeles & 12,6 & 12,1 & 15,0 & 318 \\
\hline Madrid & 10,2 & 10,8 & 37,5 & 379 \\
\hline Manila & 22,5 & 11,5 & 60,0 & 1.794 \\
\hline Medellín & 26,9 & 4,3 & 79,2 & 1.559 \\
\hline México & 26,2 & 11,5 & 52,0 & 705 \\
\hline Monterrey & 25,9 & 10,8 & 65,0 & 508 \\
\hline Montevideo & 20,3 & 16,2 & 59,0 & 1.032 \\
\hline Quito & 23,3 & 12,9 & 77,0 & 838 \\
\hline San Juan (AMSJ) & 9,3 & 20,9 & 41,0 & 1.520 \\
\hline Santiago de Chile & 26,0 & 7,6 & 36,0 & 223 \\
\hline Sao Paulo & 20,3 & 9,0 & 80,0 & 1.432 \\
\hline Tokio & 13,0 & 20,9 & 81,0 & 1.537 \\
\hline Promedio & 22,6 & 11,7 & 56,8 & 904 \\
\hline Medellín vs media & 1,19 & 0,37 & 1,39 & 1,72 \\
\hline
\end{tabular}

Varios análisis de correlación de los parámetros característicos de las ciudades, fueron realizados. Las figuras 5 a 12 muestran los casos que mostraron mayores correlaciones, para el $\mathrm{PM}_{2.5}$ y para el ozono. La tabla 6 muestra los factores de correlación lineal para las variables que se consideran significativas en el análisis realizado. Se observa que en general las correlaciones son bajas, lo cual tiene que ver con la complejidad del comportamiento, que evidentemente no se puede reducir al impacto lineal de una sola variable. Las mejores correlaciones se encuentran para el $\mathrm{PM}_{2.5}$.

Es interesante observar en las figuras 5 y 8 como el ingreso per cápita y la densidad de propiedad vehicular (carros por mil habitantes) están correlacionados significativa y negativamente con las concentraciones de $\mathrm{PM}_{2.5}$, dando entender que las ciudades de los países avanzados, a pesar de tener mayor actividad económica y mayor cantidad de carros por mil habitantes, tienden a tener menores concentraciones de $\mathrm{PM}_{2.5}$ que las ciudades de países en desarrollo. Ello posiblemente se debe a que cuentan con mecanismos de control de emisiones más efectivos, no solamente vehiculares, sino también de las otras fuentes de emisión. Lo anterior significa que las ciudades como Medellín pueden avanzar en sus planes de desarrollo económico sin que ello necesariamente implique un deterioro de su calidad ambiental, siempre y cuando avancen también en sus aspectos culturales, de organización y de sofisticación de los controles de emisiones asociados con los desarrollos que va alcanzando en equipos, producción, infraestructura y transportes. Por otra parte, se aprecia en la figura 6 que las ciudades con alta accidentabilidad (que en cierta forma es un indicador de subdesarrollo) tienden a mostrar mayores concentraciones de PM2.5, lo mismo que las más densamente pobladas (figura7), lo cual parece implicar que existe en estas ciudades menor espacio físico disponible en el entorno urbano para disipar la contaminación producida por las fuentes. 


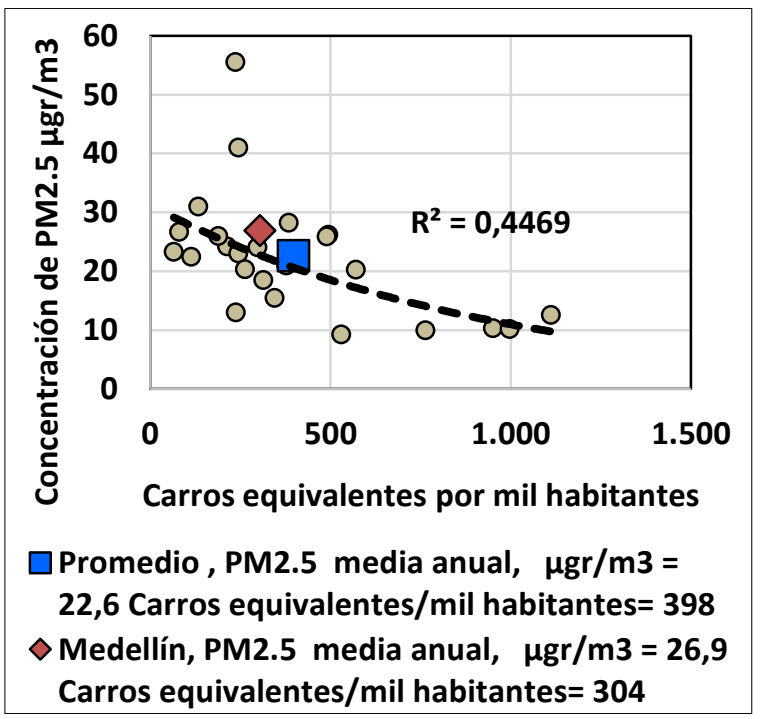

Fig. 5.

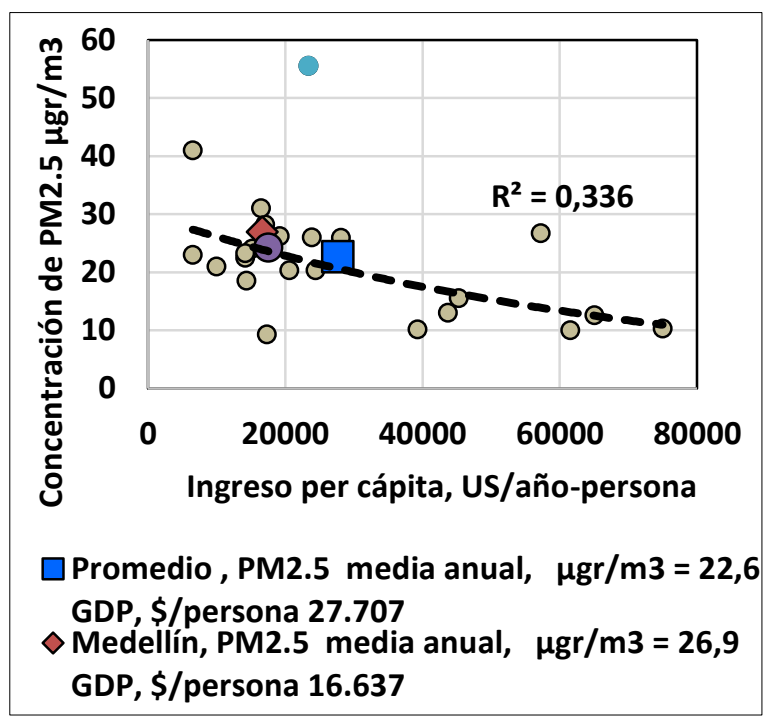

Fig.7

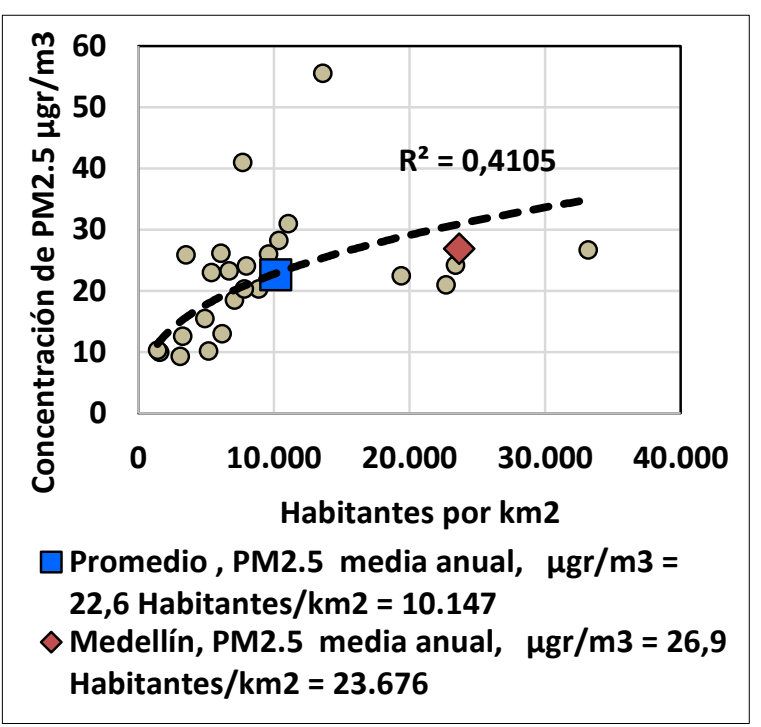

Fig. 6

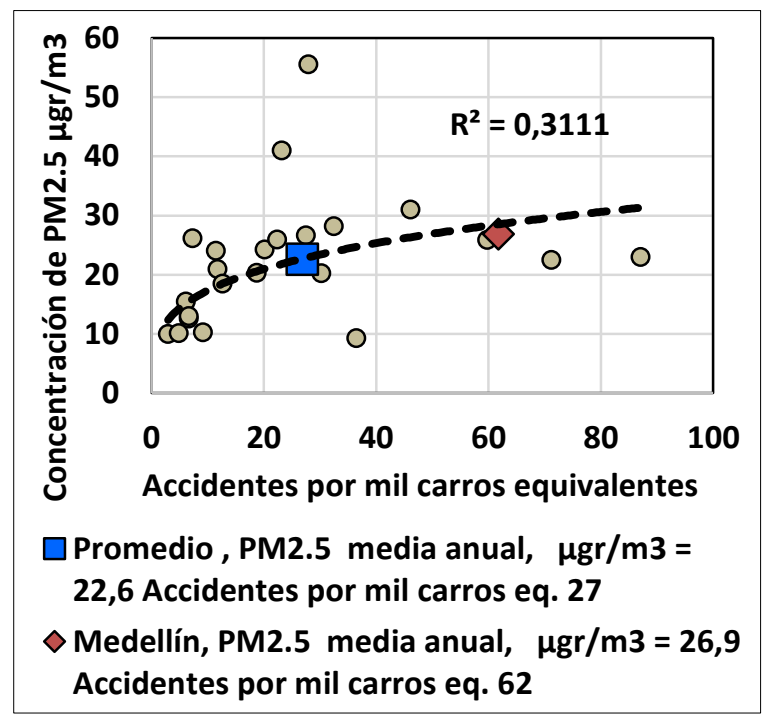

Fig. 8

Fig. 5, 6, 7, 8. Correlaciones más significativas encontradas. 


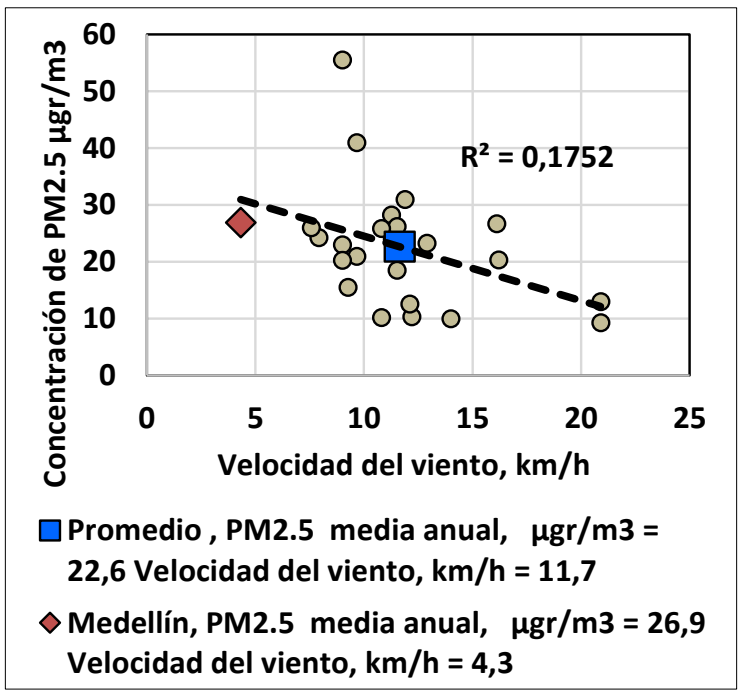

Fig. 9

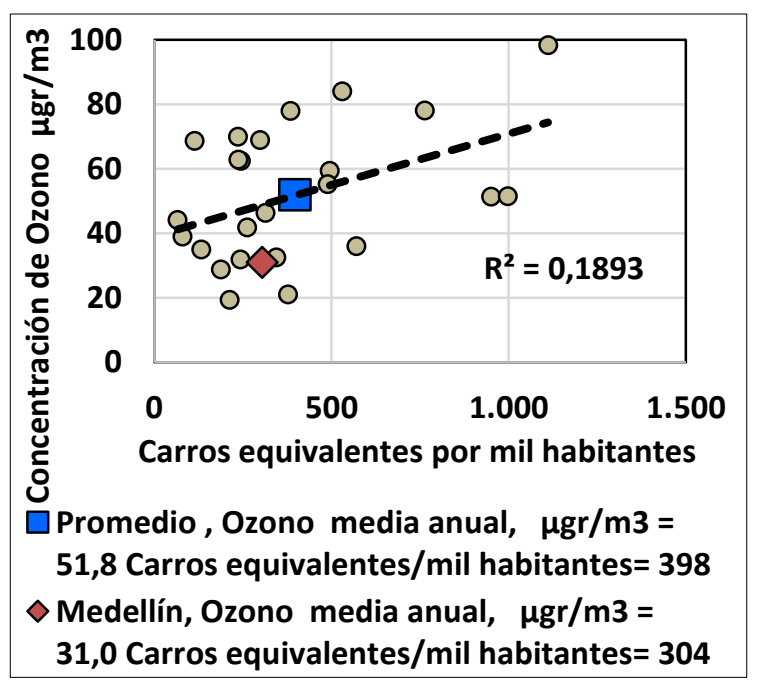

Fig. 11

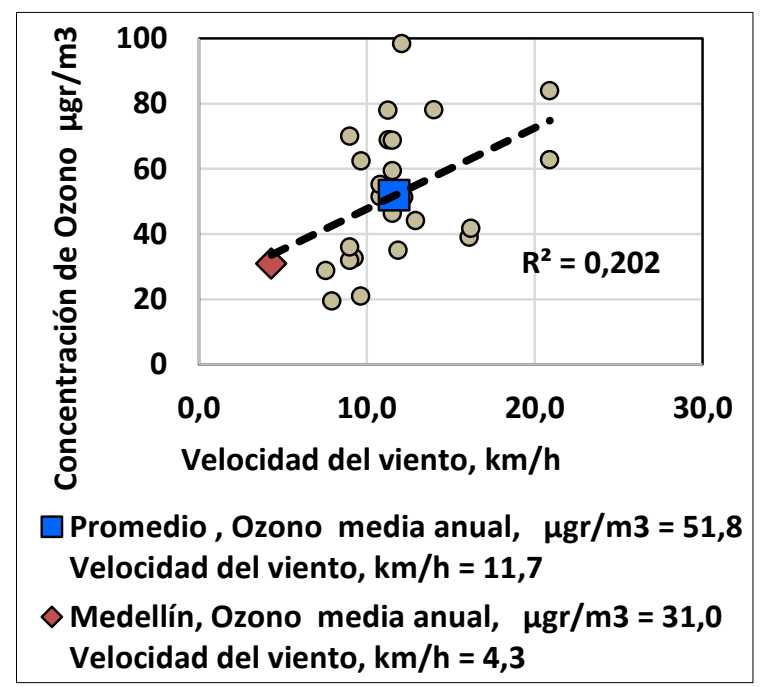

Fig. 10

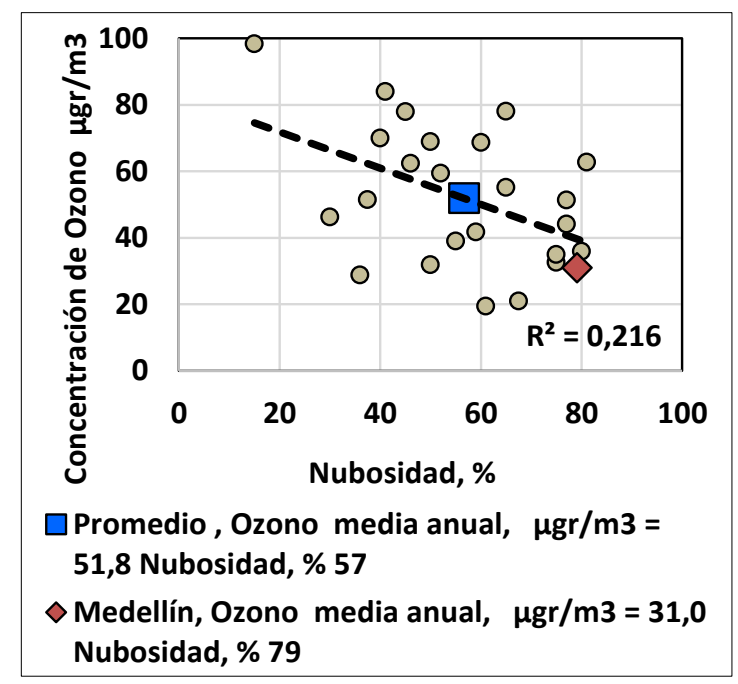

Fig. 12

Figuras $9,10,11,12$. Otras correlaciones encontradas

La figura 9 muestra que, a mayor ventilación, menores tienden a ser las concentraciones de PM2.5, algo que tiene pleno sentido desde el punto de vista de la dispersión. No se observa la misma influencia positiva de la ventilación para el caso del ozono (figura 10), probablemente porque tienen gran importancia en el caso del ozono los fenómenos de brillo solar (o inversamente de nubosidad) como se observa en la figura 12, los cuales a su vez tienden a correlacionar negativamente con la ventilación (ver los datos de la tabla 5 en la cual con la excepción de Tokio se observa esta tendencia). Por otra parte, se observa en la figura 11 que el ozono tiende a ser mayor en las ciudades con mayor actividad vehicular, lo que es de esperar dada la clara relación que hay entre emisiones vehiculares y formación de ozono [14].

La tabla 6 muestra los factores de correlación lineales que se obtienen al cruzar en forma apareada las diversas variables de calidad con las variables de influencia consideradas. 
Tabla 6. Factores de correlación encontrados al cruzar los datos de calidad anual del aire con los diferentes parámetros estudiados

\begin{tabular}{|c|c|c|c|c|}
\hline Variable considerada & unidades & $\begin{array}{l}\text { PM2.5, } \\
\mu g r / m^{3}\end{array}$ & $\begin{array}{l}\text { Ozono, } \\
\mu \mathrm{gr} / \mathrm{m}^{3}\end{array}$ & $\begin{array}{l}\mathrm{NO2,} \\
\mu \mathrm{gr} / \mathrm{m}^{3}\end{array}$ \\
\hline PM2.5 & $\mu \mathrm{gr} / \mathrm{m}^{3}$ & 1,000 & $-0,093$ & 0,295 \\
\hline Ozono & $\mu \mathrm{gr} / \mathrm{m}^{3}$ & $-0,093$ & 1,000 & 0,301 \\
\hline $\mathrm{NO}_{2}$ & $\mu \mathrm{gr} / \mathrm{m}^{3}$ & 0,295 & 0,301 & 1,000 \\
\hline Factor densidad poblacional & Habitantes $/ \mathrm{km}^{2}$ & 0,361 & $-0,446$ & 0,300 \\
\hline Factor propiedad vehicular & $\begin{array}{l}\text { Carros equivalentes/mil } \\
\text { habitantes }\end{array}$ & $-0,548$ & 0,435 & 0,105 \\
\hline $\begin{array}{l}\text { Factor densidad vehicular } \\
\text { Factor ocupación vehicular } \\
\text { Factor velocidad viento }\end{array}$ & $\begin{array}{c}\text { Carros equivalentes } / \mathrm{km}^{2} \\
\% \text { área urbana } \\
\mathrm{Km} / \mathrm{hr}\end{array}$ & $\begin{array}{r}0,066 \\
0,060 \\
-0,419\end{array}$ & $\begin{array}{c}-0,297 \\
-0,329 \\
0,449\end{array}$ & $\begin{array}{c}0,209 \\
0,151 \\
-0,005\end{array}$ \\
\hline $\begin{array}{l}\text { Factor de desnivel entre zona } \\
\text { vecina y urbana }\end{array}$ & $\mathrm{m}$ & 0,261 & $-0,018$ & 0,299 \\
\hline Factor de Ingreso Per Cápita & US \$/habitante & $-0,476$ & 0,286 & 0,207 \\
\hline $\begin{array}{l}\text { Factor de accidentabilidad } \\
\text { poblacional }\end{array}$ & $\begin{array}{c}\text { Accidentes por cada mil } \\
\text { habitantes }\end{array}$ & 0,024 & 0,040 & $-0,074$ \\
\hline $\begin{array}{l}\text { Factor de accidentabilidad } \\
\text { vehicular }\end{array}$ & $\begin{array}{l}\text { Accidentes por cada mil } \\
\text { carros equivalentes }\end{array}$ & 0,293 & $-0,166$ & $-0,114$ \\
\hline $\begin{array}{l}\text { Factor de Nubosidad } \\
\text { Factor de Precipitación }\end{array}$ & $\begin{array}{c}\% \\
\mathrm{~mm}\end{array}$ & $\begin{array}{l}-0,101 \\
-0,124\end{array}$ & $\begin{array}{l}-0,465 \\
-0,137\end{array}$ & $\begin{array}{r}-0,361 \\
0,018\end{array}$ \\
\hline
\end{tabular}

Los factores de correlación resaltados son aquellos cuyo valor absoluto es mayor de 0.35 y se pueden considerar como los más significativos.

Dado los resultados de la tabla 6, se considera que hay suficientes bases para establecer un modelo conjunto de correlación, que considere las diversas variables en una única expresión de la forma siguiente:

$$
I A_{i k}=C b+\sum F_{i j} N_{j k}
$$

Indicador modelado para cada parámetro ambiental i y para cada ciudad $\mathrm{k}$ (IAik) = Constante base $(\mathrm{Cb})$ + Sumatoria de Factores de influencia para cada indicador ambiental i de cada parámetro j de cada ciudad k (Fijk) $x$ valor normalizado de cada parámetro j para cada ciudad k, Nj.

Los valores normalizados se obtuvieron dividiendo el parámetro para cada ciudad por el valor promedio del mismo para la muestra de las ciudades. Se encontraron soluciones para cada indicador ambiental y para el indicador global ambiental mediante el empleo de la rutina solver de Excel, sujeta a la restricción de que el valor promedio de los valores absolutos de los errores para cada ciudad estuviera por debajo de un valor señalado, cercano al $20 \%$. Con la rutina se escogió la solución que diera el mínimo error. La tabla 7 muestra los resultados obtenidos.

Tabla 7. Factores de influencia en la modelación

\begin{tabular}{lcccccc}
\hline Factor influyente & Unidades & Medio & PM2.5 & $\mathbf{N O}_{2}$ & Ozono & IA \\
\hline Límite permisible & $\mu \mathrm{gr} / \mathrm{m}^{3}$ & & 25 & 100 & 60 & 1,00 \\
$\begin{array}{l}\text { Concentración media anual } \\
\text { reportada }\end{array}$ & $\mu \mathrm{gr} / \mathrm{m}^{3}$ & & 22,6 & 38,9 & 51,8 & \\
$\begin{array}{l}\text { Concentración media anual } \\
\text { modelo }\end{array}$ & $\mu \mathrm{gr} / \mathrm{m}^{3}$ & & 20,0 & 35,3 & 42,5 & \\
$\begin{array}{l}\text { Error medio en la modelación } \\
\text { Índices ambientales medios }\end{array}$ & $\%$ & & 11,7 & 9,3 & 18,1 & 7,4 \\
reportados & & 0,905 & 0,389 & 0,864 & 0,766 \\
$\begin{array}{l}\text { Índices ambientales medios } \\
\text { modelados }\end{array}$ & & & 0,799 & 0,353 & 0,708 & 0,709 \\
$\begin{array}{l}\text { Constante base Cb } \\
\text { Concentración base }\end{array}$ & $\mu \mathrm{gr} / \mathrm{m}^{3}$ & & 0,500 & 0,288 & 0,329 & 0,500 \\
Factor densidad poblacional & Habitantes/ & 10.147 & 0,277 & 0,020 & 0,000 & 0,061 \\
\hline
\end{tabular}




\begin{tabular}{|c|c|c|c|c|c|c|}
\hline Factor influyente & Unidades & Medio & PM2.5 & $\mathrm{NO}_{2}$ & Ozono & IA \\
\hline & $\mathrm{km}^{2}$ & & & & & \\
\hline & Carros & & & & & \\
\hline Factor propiedad vehicular & $\begin{array}{l}\text { equivalentes/ } \\
\text { habitante }\end{array}$ & 398 & $-0,016$ & 0,039 & 0,135 & 0,000 \\
\hline Factor densidad vehicular & $\begin{array}{c}\text { Carros } \\
\text { equivalentes/ } \\
\mathrm{km}^{2}\end{array}$ & 2.877 & 0,000 & 0,000 & 0,000 & 0,000 \\
\hline Factor ocupación vehicular & \% área urbana & 1,95 & 0,000 & 0,000 & 0,000 & 0,000 \\
\hline Accidentes totales/habitantes & $\begin{array}{l}\text { Accidentes por } \\
1000 \text { habitantes }\end{array}$ & 8,17 & 0,000 & 0,000 & 0,036 & 0,000 \\
\hline accidentabilidad & $\begin{array}{c}\text { Accidentes por } \\
1000 \text { carros } \\
\text { equivalentes }\end{array}$ & 26,89 & 0,015 & 0,000 & 0,012 & 0,084 \\
\hline Factor velocidad viento & $\mathrm{Km} / \mathrm{hr}$ & 11,7 & 0,000 & 0,000 & 0,300 & 0,000 \\
\hline $\begin{array}{l}\text { Factor de desnivel entre zona } \\
\text { vecina y urbana }\end{array}$ & $\mathrm{m}$ & 125 & 0,040 & 0,033 & 0,014 & 0,073 \\
\hline Factor de Ingreso Per Cápita & US \$/habitante & 27.707 & $-0,114$ & 0,025 & 0,128 & 0,000 \\
\hline Factor de Nubosidad & $\%$ & 57 & 0,200 & $-0,097$ & $-0,173$ & $-0,008$ \\
\hline Factor de Precipitación & $\mathrm{mm}$ & 904 & $-0,102$ & 0,045 & $-0,074$ & 0,000 \\
\hline
\end{tabular}

Las figuras 13 a 16 muestran en forma gráfica las modelaciones obtenidas aplicando los factores de influencia de la tabla 7. En tales figuras se comparan los resultados de la modelación con los valores encontrados en las bases de datos analizadas para cada ciudad. Es evidente que se han encontrado ajustes bastante razonables, de manera que el modelo puede contribuir a entender tendencias e impactos derivados de acciones y de sus relaciones con las variables demográficas, climáticas y geográficas.

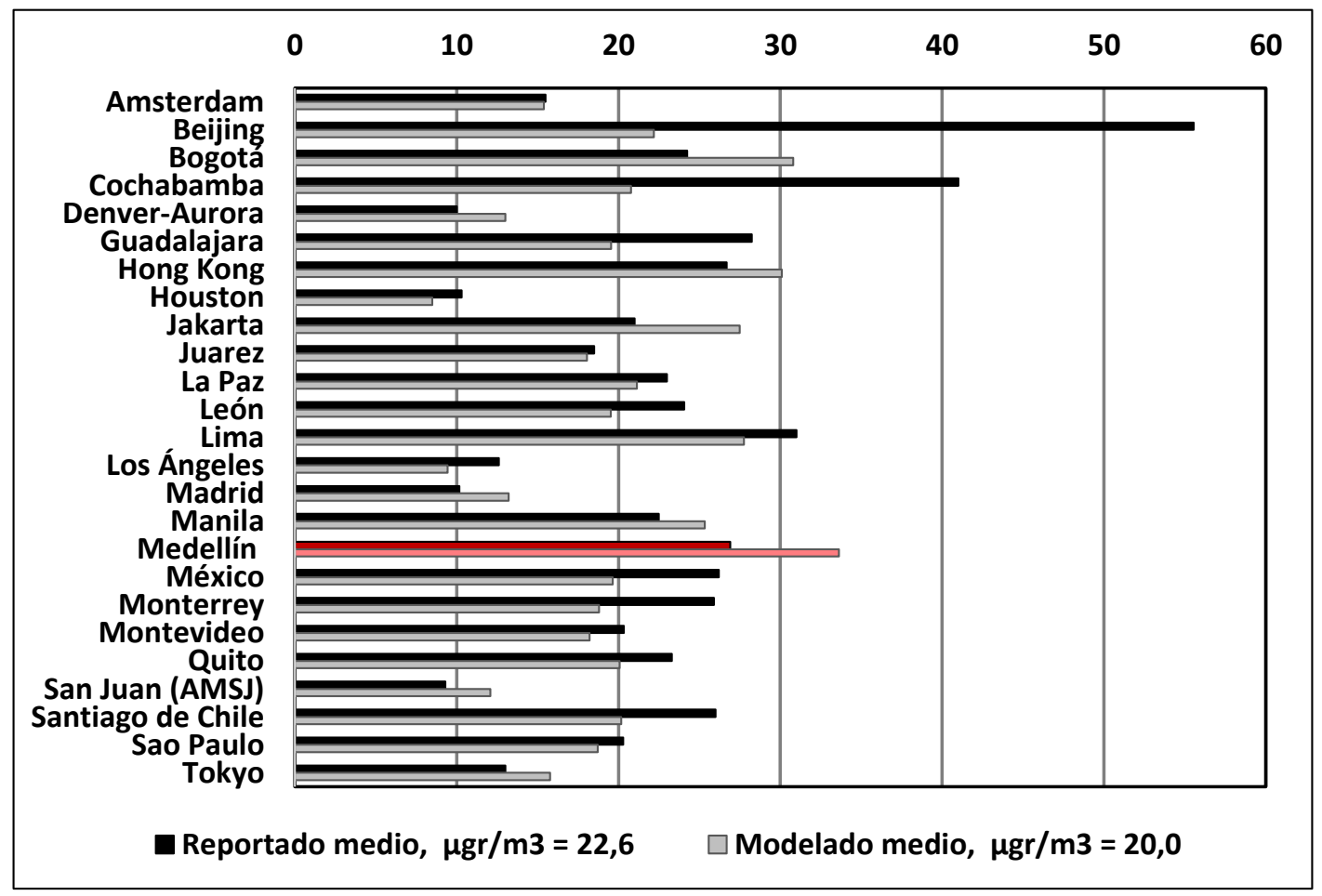

Fig. 13. Resultados reportados y modelados para el $\mathrm{PM}_{2.5}$ 


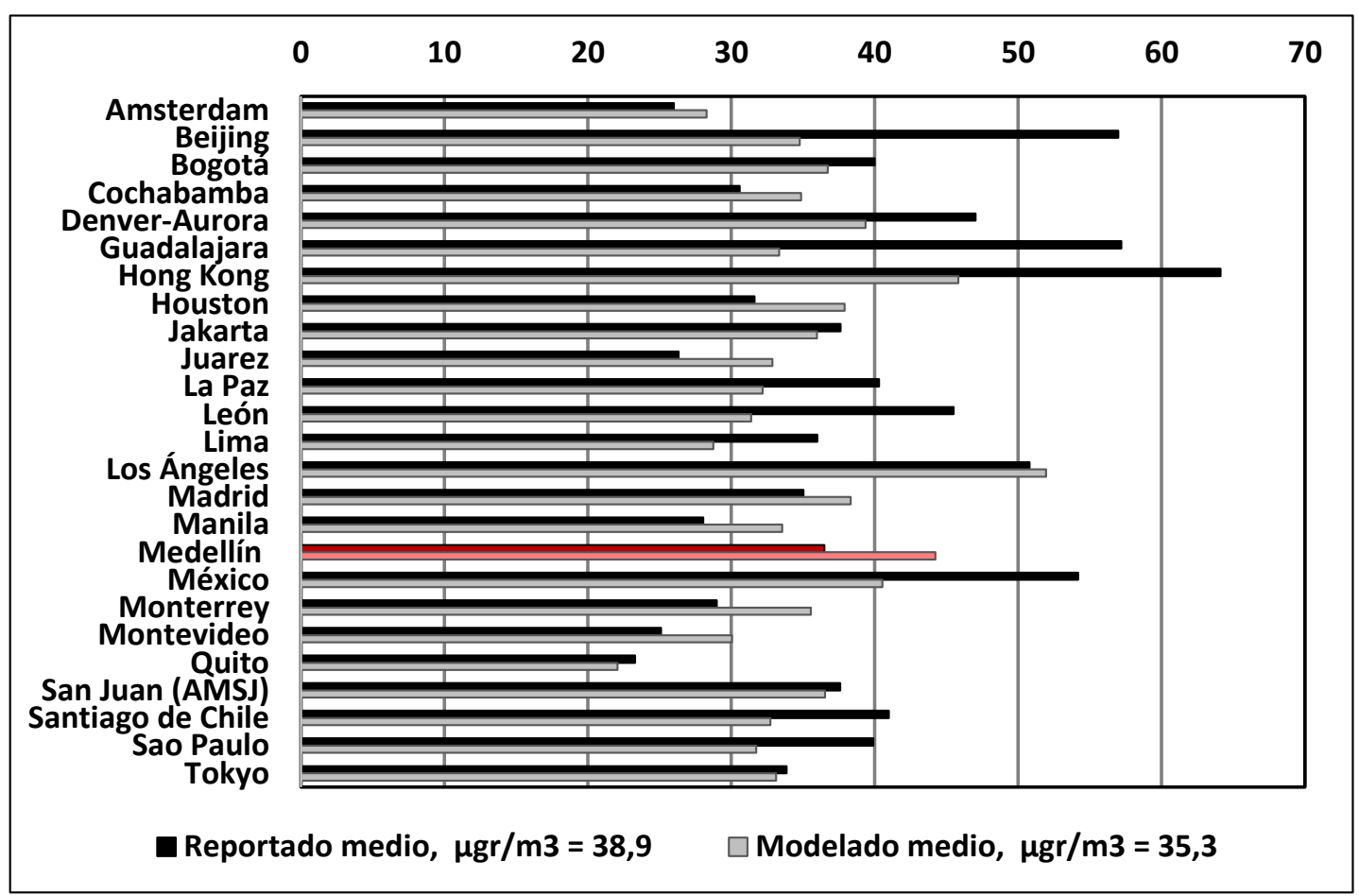

Fig. 14. Resultados reportados y modelados para el $\mathrm{NO}_{2}$

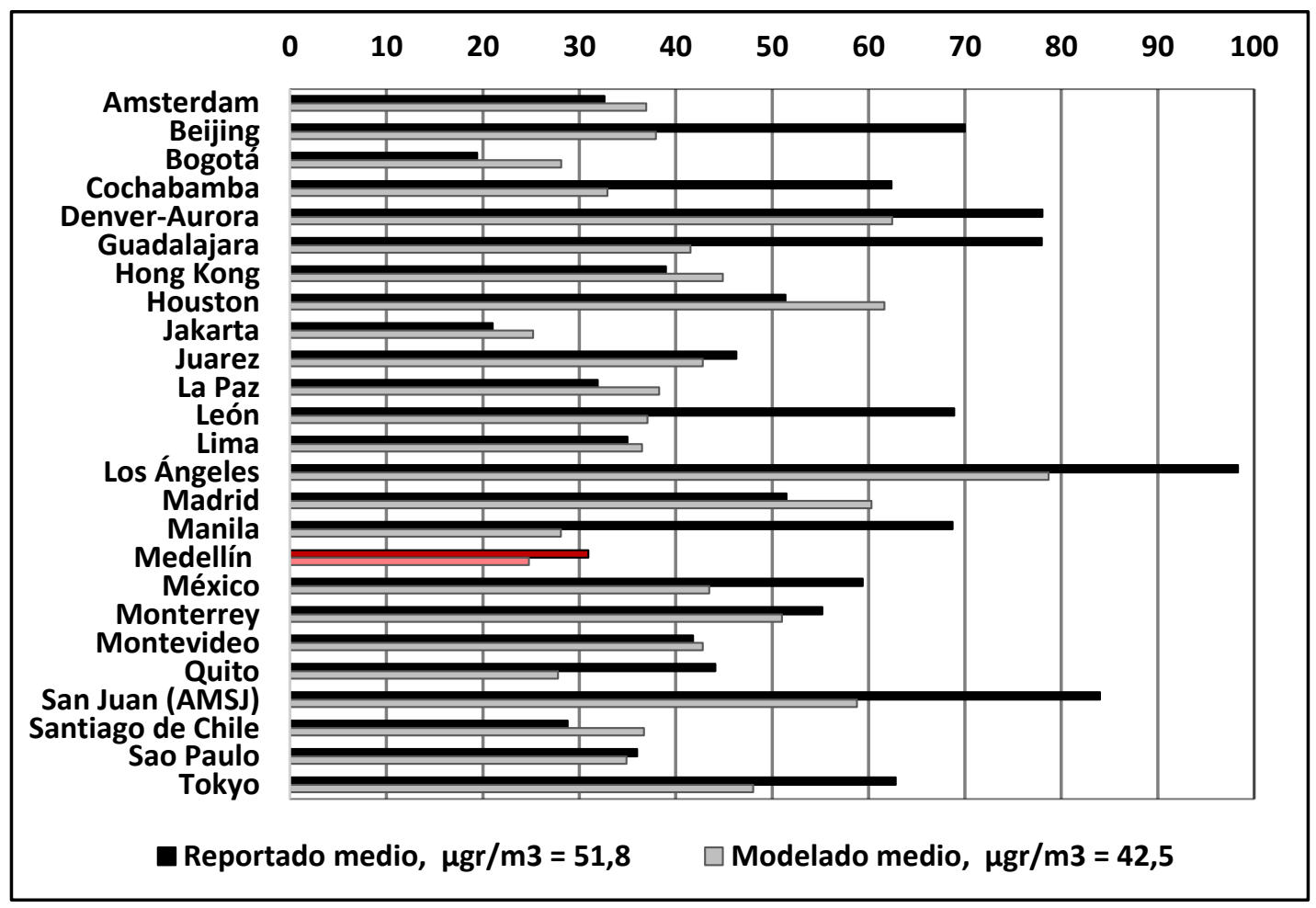

Fig. 15. Resultados reportados y modelados para el Ozono 


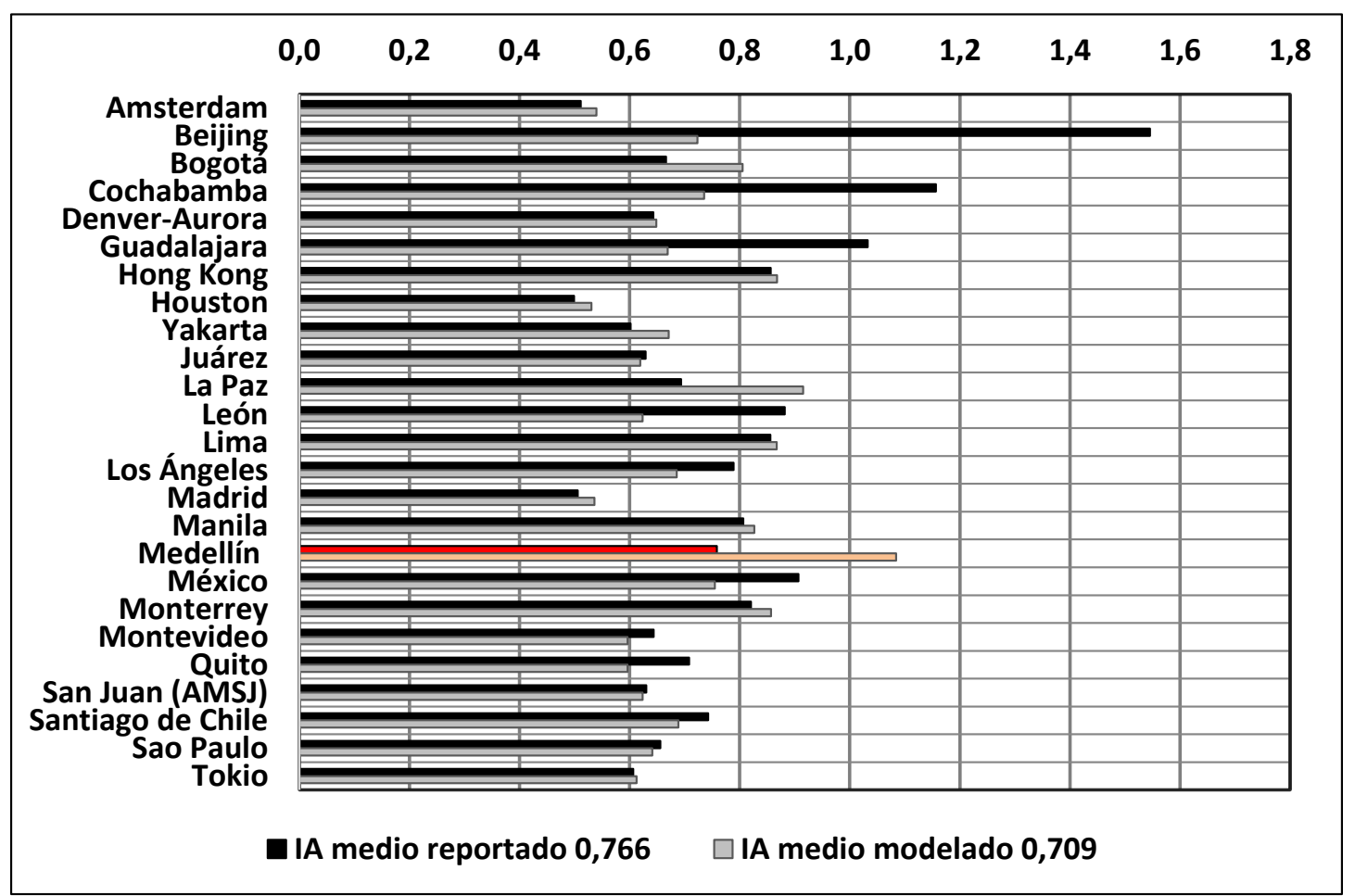

Fig. 16. Resultados reportados y modelados para el Índice combinado IA

Las figuras 17, 18 y 19 muestran los valores reportados y modelados para el índice de contaminación ambiental compuesto IA como función de los tres parámetros más influyentes

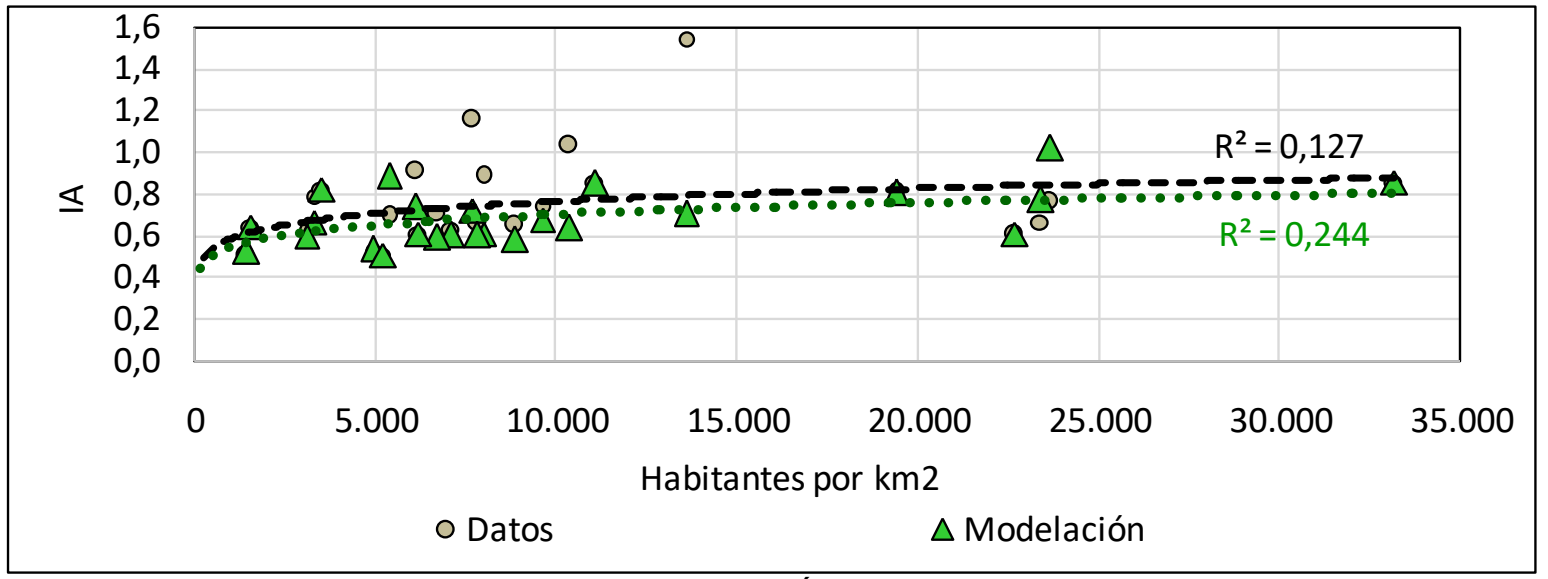

Fig. 17. Resultados reportados y modelados del Índice combinado IA vs densidad poblacional 


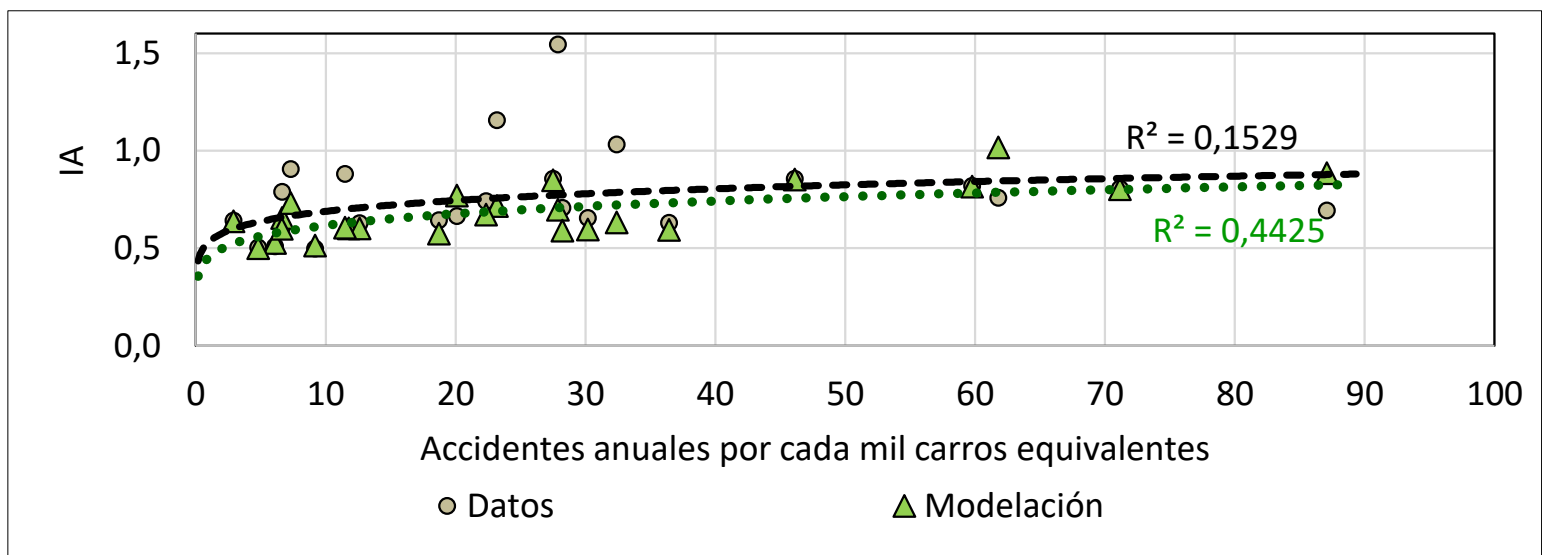

Fig. 18. Resultados reportados y modelados para el Índice combinado IA vs accidentabilidad vehicular

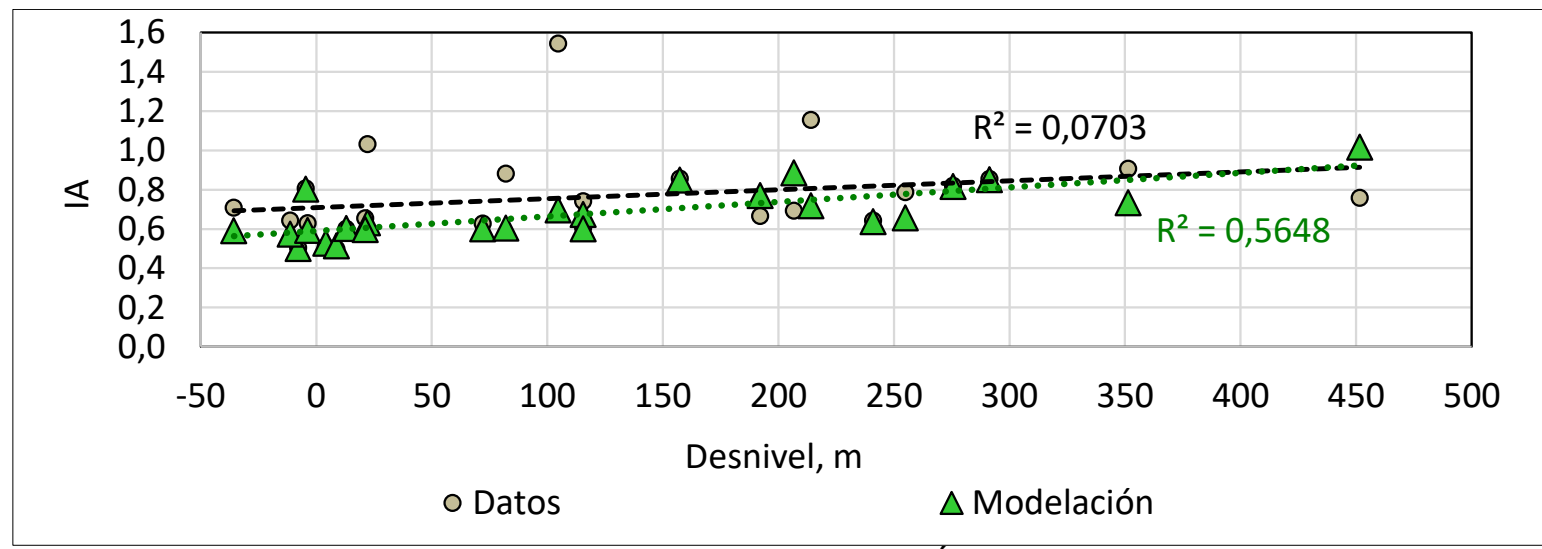

Fig. 19. Resultados reportados y modelados para el Índice combinado IA vs desnivel

\section{CONCLUSION}

Se puede concluir que es factible aproximarse a una modelación generalizada de ciudades desde el punto de vista de la calidad ambiental con base en una serie de parámetros característicos.

En general se ha logrado una buena predicción para casi todas las ciudades, con las claras excepciones de las ciudades de Beijing y Cochabamba (en las cuales los niveles reales son bastante mayores que los predichos por la modelación). En el primer caso ello puede deberse a la existencia de grandes fuentes industriales o energéticas no controladas suficientemente (como es el caso del empleo masivo del carbón) o a datos de actividad vehicular 0 de accidentabilidad no reportados bajo las mismas bases de otras ciudades de la muestra, o la existencia de una contaminación de fondo mayor, dado que muchas otras ciudades de China presentan altos niveles de contaminación [15]. En el caso de Cochabamba puede contribuir su topografía compleja, la falta de mayores controles y la existencia de prácticas culturales que aumenten las emisiones vehiculares.

Se ha encontrado que los siguientes parámetros influyen sobre los indicadores de la calidad del aire, de una manera que indudablemente es compleja, pero que de todas formas indica cierta linealidad en sus influencias. En los comentarios siguientes efecto positivo significa que el parámetro está relacionado con tendencias a aumentos de las concentraciones (impacto negativo desde lo ambiental) y efecto negativo, con tendencias a disminuciones (efecto positivo desde lo ambiental). No se pretende señalar efectos causales, sino tendencias. 
La densidad poblacional muestra impacto claro sobre el $\mathrm{PM}_{2.5}$, ligero sobre el $\mathrm{NO}_{2}$ y algo mayor sobre la calidad combinada. Afecta negativamente la calidad.

La intensidad de la propiedad vehicular está ligeramente y negativamente relacionada con el $\mathrm{PM}_{2.5}$ y ligera y positivamente con el $\mathrm{NO}_{2}$ y más claramente positivamente con el ozono. No se aprecia que sea influyente sobre la calidad combinada.

No se encontró una clara influencia de la densidad vehicular ni de la ocupación vehicular del área urbana.

La accidentabilidad por habitantes mostró ligera influencia positiva sobre el ozono. La accidentabilidad por vehículos mostró ligera influencia positiva sobre el $\mathrm{PM}_{2.5}$, el ozono y la calidad combinada. Es decir, la accidentabilidad creciente se la encontró asociada impactos negativos sobre la calidad.

La velocidad del viento mostró ligera influencia positiva sobre el ozono. No se apreció efecto en los aspectos ambientales combinados.

La diferencia de nivel entre la zona vecina y la zona urbana muestra efecto de aumento sobre todos los tres contaminantes y sobre el índice combinado. Es decir, es menor la calidad del aire para las ciudades rodeadas de montañas.

Las ciudades de alto Ingreso Per Cápita mostraron mejor calidad del aire en cuanto al $\mathrm{PM}_{2.5}$ e inferior calidad en cuanto al $\mathrm{NO}_{2}$ y el ozono. Este efecto no se nota en la calidad combinada.

La nubosidad influye para aumentar las concentraciones de $\mathrm{PM}_{2.5}$, pero tiene un efecto contrario sobre las de $\mathrm{NO}_{2}$ y Ozono. El impacto global es ligeramente positivo sobre la calidad ambiental.

A mayor precipitación se notaron menores concentraciones de $\mathrm{PM}_{2.5}$ y de ozono, pero mayores del $\mathrm{NO}_{2}$. No se nota el impacto en la calidad global.

Se advierte que muchos de los parámetros tienen influencias opuestas sobre algunos de los contaminantes.
Parece evidente que las ciudades desarrolladas se han aproximado a una situación ambiental buena para el caso del $\mathrm{PM}_{2.5}$, a pesar de que son ciudades con mayor actividad económica y mayor intensidad del uso de los vehículos. Las ciudades en desarrollo muestran las mayores concentraciones de $\mathrm{PM}_{2.5}$. Tales ciudades muestran en general altas densidades poblacionales y algunas están situadas en valles y zonas de alto desnivel. Tienen estas ciudades el desafío de desarrollar su crecimiento estableciendo estándares de alta calidad en sus emisiones y en sus buenas prácticas de conducción y cultura ciudadana. Hay que anotar que son las ciudades de mayor accidentabilidad, aspecto que también se vio correlacionado con calidades inferiores del aire.

Otro aspecto a considerar es que tales ciudades en desarrollo tienden a mostrar altos niveles de congestión, como se refleja en los porcentajes de ocupaciones de los vehículos con relación a sus áreas urbanas, los cuales son muy altos, particularmente en el caso de Medellín. Como las tendencias son crecientes, tales ciudades deben tomar decisiones apropiadas en este sentido, para evitar colapsos físicos e impactos culturales negativos. Los autores piensan que tal como ocurre con las soluciones que se dan al tema de las viviendas, habrá que pensar en sistemas elevados - subterráneos para liberar áreas y en un claro refuerzo a los sistemas masivos de transporte.

Distinta es la situación para el $\mathrm{NO}_{2}$ y para el ozono, sustancias para las cuales se nota un impacto negativo de las actividades de alto desarrollo (ingreso per cápita y densidad vehicular) y en general mayores concentraciones para las ciudades de mayor nivel de desarrollo. Ello significa que para tales contaminantes es de esperar aumentos futuros en las ciudades de los países en desarrollo, a no ser que se trabaje en el control intenso de las fuentes móviles, que son la mayor fuente de emisiones directas e indirectas de estos contaminantes.

Hay que advertir que existen unos niveles de contaminación de fondo inherentes a las zonas urbanas, los cuales tienen que ver, en el caso del $\mathrm{PM}_{2.5}$ con las emisiones provenientes de actividades agropecuarias, fuentes naturales, quemas, y transportes globales de polvo y otras fuentes. Según el análisis de los datos, tales concentraciones de fondo son del orden de los 12 microgramos por metro cúbico (que es un valor 
muy significativo, del orden del $50 \%$ del límite permisible colombiano). Es bueno señalar que algunos estudiosos de la salud y entidades como la OMS, mencionan valores de $10 \mu \mathrm{gr} / \mathrm{m}^{3}$ como dañinos para la salud. De hecho, se ha propuesto que cualquier concentración de este contaminante, por pequeña que sea va a ser dañina, por lo cual no debería hablarse de límite permisible [16], [17]. Si este es el caso, habrá que radicalizar el control de las actividades humanas y de sus emisiones hasta extremos para los cuales todavía no se ha desarrollado la metodología, incluyendo la necesidad de proteger a las personas y a sus ambientes con sistemas de purificación y filtración individuales y colectivos.

Los valores de fondo encontrados como resultado del presente análisis son del orden de $28 \mu \mathrm{gr} / \mathrm{m}^{3}$ para el $\mathrm{NO}_{2}$ y de $20 \mu \mathrm{gr} / \mathrm{m}^{3}$ para el ozono (valores ambos significativos, del orden del $30 \%$ del límite permisible considerado en este estudio). Tienen que ver con fuentes agroindustriales de compuestos nitrógeno, con otras fuentes no urbanas, y con circulaciones universales de gases y aerosoles sus reacciones secundarias.

Los autores consideran que, para el caso de Medellín, dada la riqueza de energía eléctrica de origen renovable de la región, se debería emprender el camino hacia la conversión de los sistemas de transporte público y privado hacia sistemas basados en la electricidad. En esta forma se puede aliviar significativamente el impacto de los vehículos sobre los tres contaminantes estudiados.

Consideran también que es imperativo desarrollar buenas prácticas culturales y de conducción, para enfrentar la creciente tendencia al crecimiento del parque automotor y de motocicletas, Medellín muestra índices muy altos de ocupación de área por impacto vehicular y de accidentabilidad (de los mayores de la muestra) y manejar esto implica grandes esfuerzos culturales y educativos. Se requiere desarrollar una "cultura del buen uso de las motos" y de "conducción calmada y respetuosa" para todos los vehículos. Tal cultura debe incluir destinar presupuestos a la publicidad y la difusión de las buenas prácticas culturales y éticas de la población en todo lo que tiene que ver con el medio ambiente: compartir vehículos; planear viajes; orden, aseo y limpieza; evitar botar basuras y residuos a la calle; cumplimiento de las normas de tránsito; mantenimiento de equipos y vehículos. En todo esto hay que aprovechar los nuevos desarrollos propios de las denominadas ciudades inteligentes [18]

\section{AGRADECIMIENTOS}

Los autores agradecen el apoyo del área metropolitana del Valle de Aburrá por la cofinanciación base de la información primaria de este estudio. Igualmente, al Politécnico Colombiano Jaime Isaza Cadavid por su confianza y respaldo al Grupo GHYGAM, a HATCH INDISA S.A por el soporte técnico en las rutinas de análisis y de cálculo que fortalecieron los hallazgos para la comparabilidad de la calidad del aire de Medellín con las demás ciudades del mundo, basada en datos validables y trazables.

\section{REFERENCIAS BIBLIOGRÁFICAS}

[1] Green, J. Sánchez, S. La Calidad del Aire en América Latina: Una Visión Panorámica, Clean Air Institute, 2013.

[2] WHO's Urban Ambient Air Pollution database- Update 2016.

[3] United States Environmental Protection Agency. Air Data: Air Quality Data Collected at Outdoor Monitors across the US. Disponible en: https://www.epa.gov/outdoorair-quality-data [Consultado el 20 de noviembre de 2016]

[4] Dirección general de sostenibilidad y planificación de la movilidad. La Calidad del Aire, Madrid 2014.

[5] European Environment Agency, Air pollution fact sheet 2014, Netherlands, 2014.

[6] INFORME DE CALIDAD DE AIRE, Informe anual Servicio Evaluación de la Calidad y Control Ambiental, Departamento de Desarrollo Ambiental Intendencia de Montevideo, s.f., 2013

[7] Mo Dana, Guoshun Zhuanga, B, Xinxin Lia, Hairong Taoa, Yahui Zhuangc. The characteristics of carbonaceous species and their sourcesin PM2.5 in Beijing. The Center for Atmospheric Environmental Study, Beijing 
Normal University, Beijing 100875, PR China.2013.

[8] Posada, E. Gómez, M. Cadavid, A. THE IMPORTANCE OF MOTOR VEHICLE EMISSIONS ON THE PM ${ }_{2.5}$ POLLUTION OF THE CITY OF MEDELLÍN AS DEDUCTED FROM THE HOURLY VARIATIONS IN PM 2.5 CONCENTRATIONS AND FROM CHEMICAL ANALYSIS OF PM2.5 MATERIAL. Presented at Air Quality 2016, Milán, Italia, marzo de 2016.

[9] Gebhart, Kristi A. Malm, William C. Flores M. A Preliminary Look at Source-Receptor Relationships in the Texas-Mexico Border Area. Journal of the Air \& Waste Management Association, 50,858-868, 2000.

[10] Resolución 610, Ministerio de Ambiente, Vivienda y Desarrollo Territorial. República de Colombia, 2010

[11] Informe Final de Calidad del Aire en el Valle de Aburra, Convenio de Asociación No. 243 de 2012. Área Metropolitana del Valle de Aburra, 2013.

[12] Área Metropolitana del Valle de Aburra. Estudios de calidad del aire. Disponible en:http://www.metropol.gov.co/CalidadAire/P aginas/bibliotecaaire3.aspx [Consultado el 20 de noviembre de 2016]

[13] PERRY J. SAMSON, Dispersión Atmospheric Transport and Dispersion of Air Pollutants Associated with Vehicular Emissions. University of Michigan. En: Air Pollution, the Automobile, and Public Health. Watson AY, Bates RR, Kennedy D, editors., Washington (DC): National Academies Press (US); 1988.

[14] J.L. Jaimes-López et al. EXPERIMENTAL STUDY OF OZONE-FORMING POTENTIAL FROM EXHAUST EMISSIONS OF VEHICLES FUELED WITH REFORMULATED GASOLINE IN MÉXICO CITY. Rev. Int. Contam. Ambient. 22 (4) 165172, 2006

[15] Greenpeace. Greenpeace: China saw average PM2.5 levels fall by $10 \%$ in 2015 , but $80 \%$ of cities still fail to meet National Air
Quality Standards. Disponible en: http://www.greenpeace.org/eastasia/press/rel eases/climate-energy/2016/Q4-CityRankings-2015/ [Consultado el 20 de noviembre de 2016]

[16] WHO. Review of evidence on health aspects of air pollution - REVIHAAP, WHO Regional Office for Europe, 2013

[17] Posada E (2017) Considerations of the Impact of PM2.5 Particulate Matter a Health: The Meaning and Impact of Levels and Air Quality Limits on the Aburrá Valle Region in Colombia. J Environ Anal Toxicol 7: 467. doi: 10.4172/2161-0525.1000467

[18] Smart cities may help combat air quality issues, scientists say. Published Times of India, Apr 20, 2016 in http://timesofindia.indiatimes.com/home/scien ce 